\title{
Conditional deletion of smooth muscle Cullin-3 causes severe progressive hypertension
}

\author{
Larry N. Agbor, ${ }^{1}$ Anand R. Nair, ${ }^{1}$ Jing Wu, ${ }^{1,2}$ Ko-Ting Lu, ${ }^{1,2}$ Deborah R. Davis, ${ }^{1}$ Henry L. Keen, ${ }^{1}$ \\ Frederick W. Quelle, ${ }^{1}$ James A. McCormick, ${ }^{3}$ Jeffrey D. Singer, ${ }^{4}$ and Curt D. Sigmund ${ }^{1,2}$ \\ 'Department of Pharmacology, Roy J. and Lucille A. Carver College of Medicine, University of lowa, lowa City, lowa, \\ USA. ${ }^{2}$ Department of Physiology, Medical College of Wisconsin, Milwaukee, Wisconsin, USA. ${ }^{3}$ Division of Nephrology \& \\ Hypertension, Department of Medicine, Oregon Health \& Science University, Portland, Oregon, USA. ${ }^{4}$ Department of \\ Biology, Portland State University, Portland, Oregon, USA.
}

Patients with mutations in Cullin-3 (CUL3) exhibit severe early-onset hypertension, but the contribution of the smooth muscle remains unclear. Conditional genetic ablation of CUL3 in vascular smooth muscle (S-CUL3KO) causes progressive impairment in response to NO, rapid development of severe hypertension, and increased arterial stiffness. Loss of CUL3 in primary aortic smooth muscle cells or aorta resulted in decreased expression of the NO receptor soluble guanylate cyclase (sCC), a marked reduction in cGMP production, and impaired vasodilation to cGMP analogs. Vasodilation responses to a selective large-conductance $\mathrm{Ca}^{2+}$-activated $\mathrm{K}^{+}$channel activator were normal, suggesting that downstream signals that promote smooth muscle-dependent relaxation remained intact. We conclude that smooth muscle-specific CUL3 ablation impairs both cGMP production and cGMP responses and that loss of CUL3 function selectively in smooth muscle is sufficient to cause severe hypertension by interfering with the NO/sGC/cCMP pathway. Our study provides evidence that vascular smooth muscle CUL3 is a major regulator of BP. CUL3 mutations cause severe vascular dysfunction, arterial stiffness, and hypertension due to defects in vascular smooth muscle.

Conflict of interest: The authors have declared that no conflict of interest exists.

Copyright: (c) 2019 American Society for Clinical Investigation

Submitted: April 23, 2019

Accepted: June 5, 2019

Published: July 25, 2019.

Reference information: /Cl Insight. 2019;4(14):e129793. https://doi. org/10.1172/ji.insight.129793.

\section{Introduction}

Hypertension continues to be the major risk factor for cardiovascular diseases, including stroke, myocardial infarction, and renal failure (1). Hypertension afflicts more than a billion people worldwide, and this number is expected to increase in response to the release of the American College of Cardiology and American Heart Association guidelines on the prevention of hypertension in adults, which has defined a lower BP threshold for hypertension and treatment (2). While there is a large number of individuals with undiagnosed hypertension, there also are populations of hypertensive patients for whom current treatment regimens remain ineffective (3). Thus, there remains a need for the discovery of novel pathways that regulate $\mathrm{BP}$ in order to develop new ways to prevent and treat hypertension.

Cullin-3 (CUL3) was first implicated as a candidate gene in hypertension in a whole exome sequencing study of patients with familial hyperkalemic hypertension (FHHt), previously referred to as pseudohypoaldosteronism type II, a rare Mendelian syndrome featuring hypertension (4). The association has been replicated in other cohorts of FHHt patients (5-7). Electrolyte abnormalities characteristic of these disorders implicated a renal mechanism, which was supported by the identification of with-no-lysine $[\mathrm{K}]$ kinase $4($ WNK4) and WNK1 as protein targets of CUL3 $(8,9)$. The potential for a broader extrarenal role for CUL3 in hypertension stemmed from our studies showing that transgenic mice expressing the human hypertension-causing mutation in PPAR $\gamma$ (P467L) selectively in vascular smooth muscle cells exhibited hypertension, in part due to a reduction in CUL3 expression and increased RhoA/Rho kinase activity (10, 11). Consistent with this, RhoA, a small GTPase controlling Rho kinase activity in vascular smooth muscle, is a CUL3 target $(12,13)$. Later, the genetic association of CUL3 and hypertension was functionally confirmed in animal models $(14,15)$. Importantly, mice carrying mutations of $C U L 3$ that cause hypertension in humans revealed that renal and vascular CUL3 may both independently regulate arterial BP $(16,17)$. 
CUL3 is a scaffold protein involved in the formation and function of the CUL3-RING E3 ubiquitin ligase (CRL) complex (18). This complex is involved in regulating protein turnover by ubiquitination and subsequent degradation of target proteins by the proteasomal pathway (19). CUL3 interacts with substrate recognition proteins, also known as BTB domain-containing adaptors, to provide target selectivity and specificity for ubiquitination by CRL3 complexes (20). In a canonical example, Keap1, a BTB domain-containing protein, regulates the ubiquitination and turnover of $\mathrm{Nrf} 2$, a transcription factor involved in mounting an antioxidant response (21). BACURD1, another BTB domain-containing protein, is the substrate adaptor for RhoA (12). We identified another BTB domain-containing protein, RhoBTB1, as a PPAR target gene in vascular smooth muscle that acts as a substrate adaptor for phosphodiesterase 5 (PDE5), a key protein regulating cGMP turnover (22). There are approximately 200 different BTB domain-containing proteins that may provide substrate specificity for a large number of protein substrates (23). That CUL3 is ubiquitously expressed suggests that the range of CUL3 target proteins may be large and complex. However, which targets are involved in regulating arterial BP remains an open question.

To elucidate whether smooth muscle CUL3 dysfunction is sufficient to promote hypertension and to better understand its contribution to BP regulation, we tested the effect of conditional genetic ablation of CUL3 specifically in vascular smooth muscle (S-CUL3KO) of mice. We show that smooth muscle-specific deletion of CUL3 is sufficient to promote progressive impairment in NO responsiveness, arterial stiffness, and hypertension. Smooth muscle-specific CUL3 deficiency resulted in a significant reduction in expression of the $\beta 1$ and $\alpha 1$ subunits of the NO receptor soluble guanylate cyclase (sGC), resulting in impaired NO-induced cGMP production. Moreover, CUL3 deficiency resulted in impaired relaxation to cGMP analogs, suggesting an impaired response to cGMP during NO-mediated vasorelaxation. Taken together, these studies show that loss of smooth muscle CUL3 is sufficient to promote progressive and severe vascular dysfunction and hypertension, and identify a CUL3/NO/sGC/cGMP regulatory pathway potentially impacting the pathogenesis of hypertension.

\section{Results}

Mice carrying a conditional CUL3 allele $\left(C U L 3^{f / f f}\right)$ were bred with mice expressing a smooth muscle-specific tamoxifen-inducible Cre recombinase (Myh11-CreER ${ }^{\mathrm{T} 2}$ ). After 2 generations of breeding, the resulting $\mathrm{Cre}^{+} / C U L 3^{\text {fl/fl}}$ mice were treated with either tamoxifen to promote smooth muscle-specific deletion of CUL3 (termed S-CUL3KO) or corn oil (vehicle) as a control (Figure 1A). Tamoxifen-treated $\mathrm{Cre}^{+} / \mathrm{CUL}^{f /+}$ mice (S-CUL3het) were also produced to test the effect of smooth muscle-specific CUL3 haploinsufficiency. Western blot analysis of protein from aorta that was cleaned of adventitia revealed efficient ablation of CUL3 protein in S-CUL3KO mice (Figure 1B; full, uncut gels available in Supplemental Material). The ablation was selective for CUL3, as there was preservation of normal levels of CUL1 and CUL5, 2 other members of the Cullin family. There was no evidence of CUL3 ablation in whole heart or kidney, consistent with a much smaller contribution of smooth muscle cells in those tissues (Figure 1C). The cell specificity of CUL3 ablation was confirmed by immunostaining in aorta (Figure 1D). CUL3 staining was evident in both endothelium and smooth muscle layers of aorta from control mice, but was absent from smooth muscle layers of aorta from S-CUL3KO mice. Notably, however, positive CUL3 expression was preserved in the endothelium of S-CUL3KO aorta.

We next examined the physiological relevance of smooth muscle CUL3 ablation 2 weeks after tamoxifen administration. We performed vascular reactivity studies in the aorta, a conduit artery, and the basilar artery, a cerebral resistance vessel that supplies the brain with nutrients and oxygen. We previously showed that the basilar artery is particularly sensitive to PPAR $\gamma$ interference (24-26). Aorta from S-CUL3KO mice exhibited severe impairment in vasorelaxation responses to the endothelium-dependent agonist acetylcholine ( $\mathrm{ACh}$ ) and to the endothelium-independent NO donor sodium nitroprusside (SNP) (Figure 2, A and B). In contrast, heterozygous and WT mice showed robust vasodilation in response to both agents, suggesting that CUL3 haploinsufficiency is not sufficient on its own to cause a defect in vasodilation. Relaxation was also impaired in response to an alternative endothelium-dependent agonist, methacholine, and the NO donor DEA NONOate (Figure 2, C and D). Consistent with data from aorta, the severely impaired response to ACh and SNP was also evident in the basilar artery (Figure 2, E and F). Interestingly, the severe impairment in vasodilation responses occurred without a change in expression of total or phosphorylated (Ser1177) endothelial NO synthase (eNOS) (Supplemental Figure 1; supplemental material available online with this article; https://doi.org/10.1172/jci.insight.129793DS1). 
Taken together, these data suggest that selective ablation of CUL3 in vascular smooth muscle causes severe impairment of NO responsiveness.

We next evaluated the time course of the NO impairment by assessing ACh and SNP responses in aorta from S-CUL3KO and control mice 3, 7, 10, 14, and 18 days after completion of tamoxifen treatment. All groups exhibited normal relaxation responses to ACh and SNP 3 and 7 days after tamoxifen treatment (Figure 3, A and B, and Supplemental Figure 2, A and B). A modest impairment in ACh- and SNP-elicited relaxation was evident in S-CUL3KO mice 10 days after tamoxifen treatment (Figure 3C and Supplemental Figure 2C). This impairment progressively intensified over time until 18 days, when the vessels were completely insensitive to ACh and SNP (Figure 3, D and E, and Supplemental Figure 2, D and E). The time course of vascular impairment largely paralleled the decrease in CUL3 protein over time (Supplemental Figure 3). The maximum relaxation throughout the time course is illustrated in Figure $3 \mathrm{~F}$ and Supplemental Figure 2F. Vascular function in S-CUL3het and control mice did not change throughout the time course. These results show that loss of CUL3 in smooth muscle (S-CUL3KO) results in a progressive impairment in vascular function, reaching maximal impairment 18 days following CUL3 ablation.

We also evaluated the effects of contractile agonists on vessels from mice with smooth muscle CUL3 ablation. Aorta from S-CUL3KO mice exhibited significantly increased contraction to $10 \mathrm{mmol} / \mathrm{L}$ potassium chloride (Figure $4 \mathrm{~A})$. The overall range of the contractile response to prostaglandin $\mathrm{F}_{2 \alpha}\left(\mathrm{PGF}_{2 \alpha}\right)$ was blunted in S-CUL3KO mice (Figure 4B). However, contraction to low concentrations of agonist was significantly enhanced (Figure 4C). A similar pattern of contraction was observed with serotonin (5-hydroxytryptamine hydrochloride [5-HT]) (Supplemental Figure 4). The time course of the contractile impairment to $\mathrm{PGF}_{2 \alpha}$ mirrored what was observed for vasodilation (Supplemental Figure 5). Unlike for $\mathrm{PGF}_{2 \alpha}$ and 5-HT, there was enhanced contraction to higher doses of ET-1 (Figure 4D). Consistent with this, levels of RhoA and the phosphorylated form of myosin light chain phosphatase subunit 1 (MYPT1) were increased in aorta from S-CUL3KO mice, suggestive of elevated RhoA/Rho kinase activity (Figure 4, E and G). Moreover, the increase in ET-1-mediated vasocontraction was RhoA/Rho kinase dependent, as it was abolished by preincubation with a specific Rho kinase inhibitor, Y27632 (Figure 4H). However, treatment with the Rho kinase inhibitor did not improve ACh-mediated relaxation in aorta or basilar arteries from S-CUL3KO mice (Figure 4, I and J). Thus, vascular smooth muscle-specific ablation of CUL3 results in a more severe vascular phenotype than the dysfunction resulting from expression of a dominant negative mutant of PPAR $\gamma$, which controls the CUL3 pathway, or of a dominant negative mutant of CUL3 selectively in vascular smooth muscle $(10,11,16)$.

Oxidative stress and inflammation are hallmarks of vascular dysfunction in hypertension. There was a significant increase in protein expression of both Nox2, a subunit of NADPH oxidase, and heme oxygenase 1 (HO-1), a Nrf2 target gene activated in response to oxidative stress, in aorta from S-CUL3KO mice (Figure 5A). There was no change in the expression of inflammatory markers including the NF- $\mathrm{kB}$ subunits p65 and p50, as well as p38 MAPK (Figure 5B). Nitrotyrosine levels were elevated in aorta from S-CUL3KO mice (Figure 5C). Despite some evidence of oxidative stress, 30-minute preincubation with the superoxide scavenger Tempol modestly improved ACh-mediated vasorelaxation in aorta from S-CUL3KO mice (Figure 5D). However, Tempol only improved ACh-mediated vasodilation to $16.7 \%$, calling into question whether the improvement was physiologically significant. Together, these results suggest that other mechanisms besides elevated RhoA and oxidative stress are involved in mediating the severity of vascular dysfunction observed in S-CUL3KO mice.

We next measured arterial BP by radiotelemetry. Similar to the onset of vascular dysfunction, there was a progressive increase in systolic BP (SBP) in S-CUL3KO mice, with levels reaching a plateau 3-4 weeks after tamoxifen treatment (Figure 6, A and B). Mean arterial pressure (MAP; Figure 6, C and D), diastolic BP (DBP; Figure 6, E and F), and pulse pressure (Figure 6G) were also significantly increased in S-CUL3KO mice. There were no changes in $\mathrm{BP}$ or pulse pressure in heterozygous or control mice. There were no significant differences in heart rate (Figure 6H) or activity (Supplemental Figure 6A) between genotypes. The hypertension in S-CUL3KO mice was associated with cardiac and renal hypertrophy (Supplemental Figure $6, \mathrm{~B}$ and C). Interestingly, the levels of total angiotensin peptides in the plasma of S-CUL3KO 2 weeks after tamoxifen treatment were unchanged from control levels (Supplemental Figure 6D). Taken together, these data suggest that smooth muscle-specific ablation of CUL3 causes severe progressive hypertension.

Elevated SBP and pulse pressure are a hallmark of and major contributing factor in the development of arterial stiffening (27). Thus, we measured pulse wave velocity (PWV) as an index of arterial stiffness. 


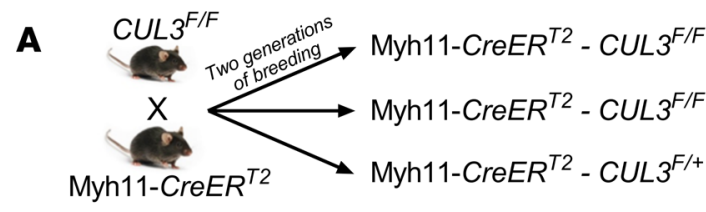

B

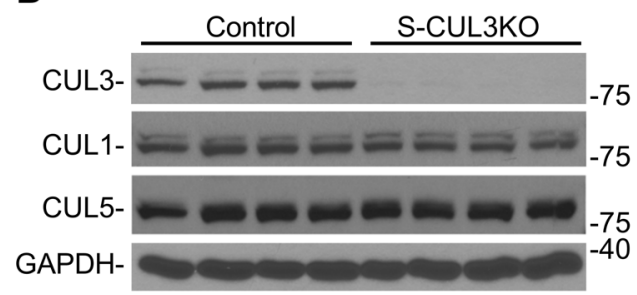

C
D

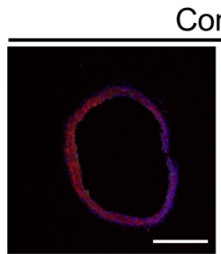

Control

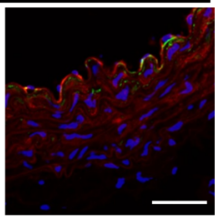

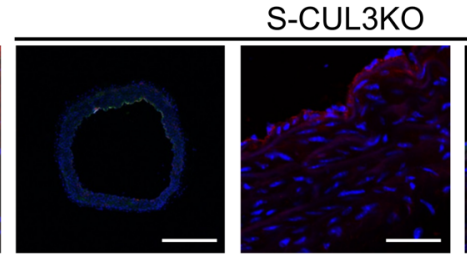

CUL3+CD31+DAPI

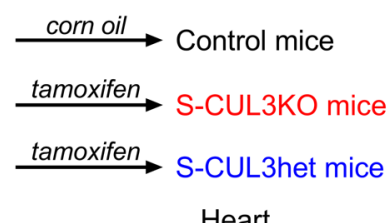

Heart
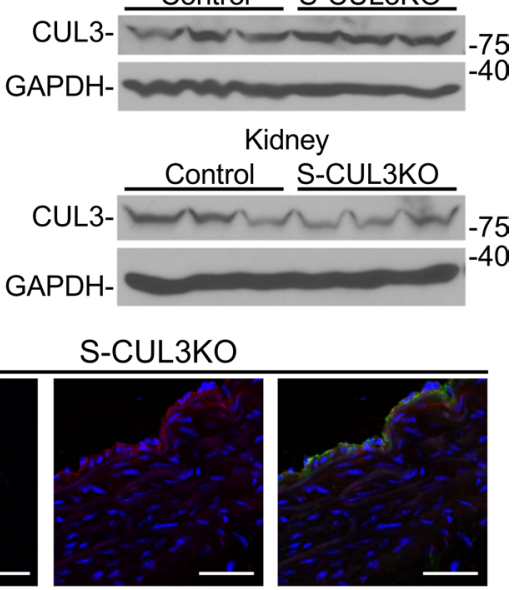

Figure 1. Smooth muscle-specific ablation of CUL3. (A) Mice carrying 2 copies of a conditional CUL3 allele were bred with mice expressing an inducible Cre recombinase driven by a smooth muscle myosin heavy chain promoter (Myh11CreER ${ }^{\mathrm{T} 2}$ ). The resultant offspring heterozygous for the CUL3 floxed allele (CUL3 ${ }^{f /+}$ ) and also expressing Myh11-CreER ${ }^{\mathrm{T} 2}$ $\left(\mathrm{Cre}^{+} / \mathrm{CUL}^{f / /+}\right)$ were backcrossed to $\mathrm{CUL}^{f^{f / f l}}$ mice. $\mathrm{Cre}^{+} / \mathrm{CUL}^{\mathrm{ff} / f l}$ mice were administered corn oil (vehicle) as controls, and $\mathrm{Cre}^{+} / \mathrm{CUL}^{\mathrm{fl/f}}$ mice or $\mathrm{Cre}^{+} / \mathrm{CUL}^{\mathrm{fl/}+}$ mice were administered tamoxifen $(75 \mathrm{mg} / \mathrm{kg})$ i.p. for 5 consecutive days to generate smooth muscle-specific CUL3 knockout (S-CUL3KO) or heterozygous mice (S-CUL3het), respectively. (B) Western blots showing expression levels of the indicated proteins in aorta of control and S-CUL3KO mice 2 weeks after administration of tamoxifen. Aortas were cleaned of perivascular adipose fat and adventitia. A representative of 5 independent experiments is shown. (C) Western blots showing expression of the indicated proteins in heart and kidney 2 weeks after tamoxifen or corn oil treatment. (D) Cross sections of aorta from control and S-CUL3KO mice 2 weeks after tamoxifen administration. Immunofluorescence for CUL3 was performed using a validated CUL3 antibody (red), and coimmunostained for the endothelial marker CD31 (green). Slides were counterstained with DAPI (blue) and visualized using a Zeiss 710 confocal microscope. Scale bars: $200 \mu \mathrm{m}$ in panels 1 and 3; $15 \mu \mathrm{m}$ in panels 2, 4, and 5 . Panel 4 is CUL3 + DAPI. Panel 5 is a merge of CUL3, CD31, and DAPI.

S-CUL3KO mice exhibited increased PWV as early as 2 weeks after tamoxifen administration (Supplemental Figure 7A), which was maintained through 4 weeks after tamoxifen treatment (Figure 7A). Consistent with this, aorta from S-CUL3KO mice exhibited significantly increased medial wall thickness in unpressurized vessels $(0 \mathrm{mmHg}$, Figure 7B), reduced outer diameter at higher pressure (Figure 7C), a downward shift of the stress-strain curve (Figure 7D), and a reduction in distensibility (Figure 7E). Similar structural changes were becoming evident 2 weeks after tamoxifen treatment (Supplemental Figure 7, B-E). While there was no difference in elastin content (Figure 7F and Supplemental Figure 7F) in the aorta between genotypes, S-CUL3KO aorta exhibited significantly increased hydroxyproline content (Figure $7 \mathrm{G})$, a biochemical measurement of collagen. Increased collagen content was observed in the adventitia of S-CUL3KO aorta 4 weeks after tamoxifen treatment, as observed by Masson's trichrome staining (Figure $7 \mathrm{H}$ ), a pathology that emerged at 2 weeks (Supplemental Figure 7, G and H). In addition, the increases in medial wall thickness observed histologically were consistent with those observed by pressure myograph (Figure 7B), indicative of medial hypertrophy in S-CUL3KO aortas. Taken together, our data suggest that loss of CUL3 in smooth muscle cells causes smooth muscle hypertrophy and impairment of arterial compliance, all contributing to aortic stiffness.

We next assessed whether expression of the NO receptor SGC was altered in S-CUL3KO aorta, because its impairment is indicative of $\mathrm{NO}$ resistance in several forms of hypertension $(28,29)$. Levels of SGC $\beta 1$ and $\alpha 1$ (sGC $\beta 1$ and sGC $\alpha 1$, respectively) subunit proteins were significantly reduced in adventitia-free aorta from S-CUL3KO mice (Figure 8A). To determine whether this was a direct effect of CUL3 ablation or a compensatory response to hypertension, we isolated and cultured primary aortic smooth muscle cells from 
A

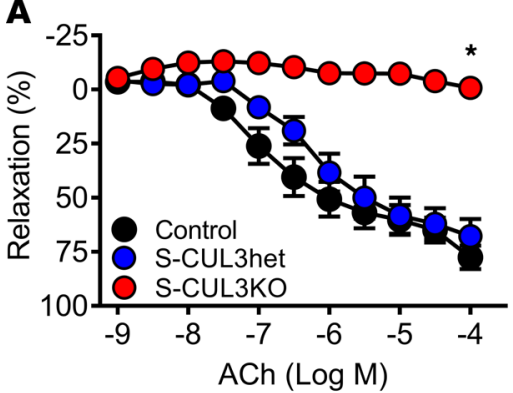

C

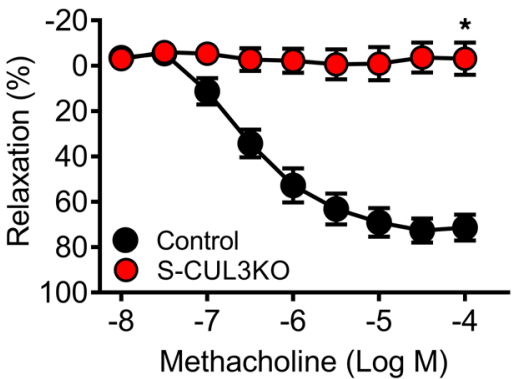

$\mathbf{E}$

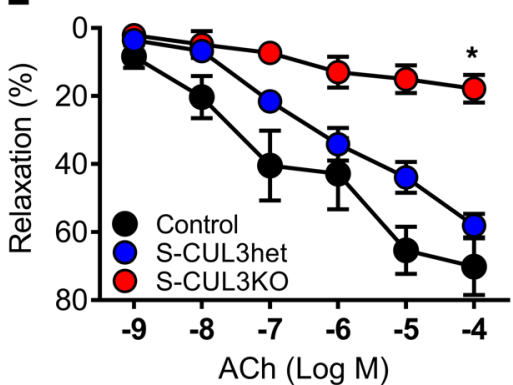

B

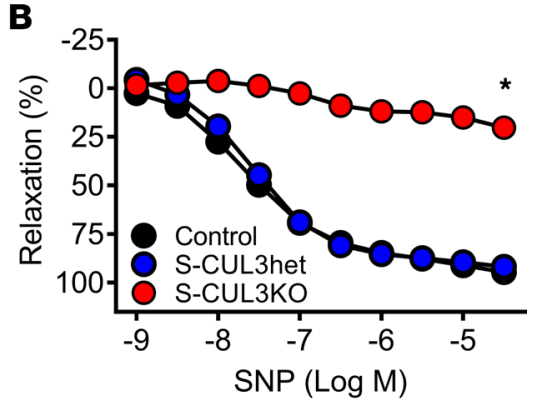

D

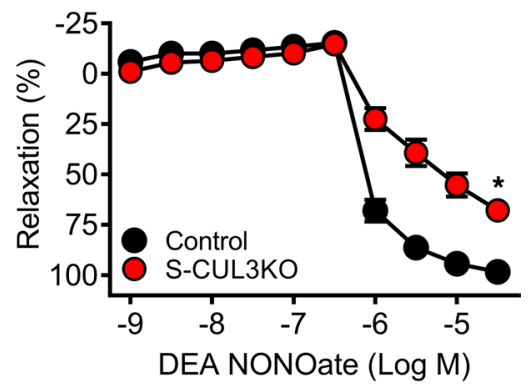

$\mathbf{F}$

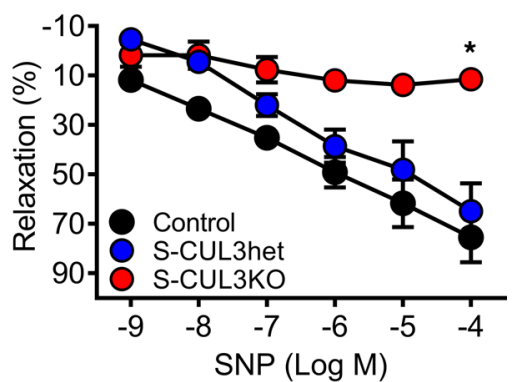

Figure 2. Vasodilation of aorta and basilar arteries. (A-D) Isometric tension was determined on aortic rings 2 weeks after control or tamoxifen administration. Dose-dependent relaxation was measured (following precontraction with prostaglandin $\mathrm{F}_{2 \alpha}\left[\mathrm{PGF}_{2 \alpha}\right]$ to $45 \%-50 \%$ of maximal PGF $2 \alpha$. Relaxation responses to acetylcholine (A, $n=8-12 /$ genotype), sodium nitroprusside (SNP; B, $n=8-12$ /genotype), methacholine (C, $n=6$ /genotype), and DEA NONOate (D, $n=6 /$ genotype) were assessed. (E and F) Basilar arteries were cannulated and pressurized to $60 \mathrm{mmHg}$ and precontracted with the thromboxane A2 mimetic (U46619) to 30\% internal diameter. Dose-dependent relaxation to acetylcholine (Ach; E, $n$ $=6-8 /$ genotype) or sodium nitroprusside (SNP; F, $n=6-8 /$ genotype) was assessed. For $\mathbf{A}-\mathbf{F}$, error bars represent mean \pm SEM. ${ }^{*} P<0.05$ by 2 -way repeated-measures ANOVA vs. other groups shown in each panel.

CUL $3^{\text {R/fl}}$ mice and infected them with adenovirus expressing Cre recombinase (AdCRE) to induce CUL3 ablation. Consistent with results from aorta, loss of CUL3 in primary aortic smooth muscle cells resulted in a significant reduction in $\mathrm{sGC} \beta 1$ and sGC $\alpha 1$ protein (Figure $8 \mathrm{~B}$ ). This was likely a transcriptional response, as the levels of mRNA encoding both subunits was markedly downregulated (Figure 8C). Interestingly, there was no change in the expression of 2 RNA binding proteins ELAV-like RNA binding protein 1 (HuR) and AU-rich element RNA-binding protein 1 (AUF1), which have both been reported to regulate the stability of sGC $\beta 1$ and sGC $\alpha 1$ mRNA (Supplemental Figure 8) (30-33). There was also no change in expression of cytochrome $b_{5}$ reductase 3 (Cyb5R3), which regulates the activity of sGC by controlling its redox state (34).

To determine whether reduced SGC expression led to a reduction in cGMP, we assessed the response to stimulation with the NO donor SNP. SNP induced robust cGMP production in control cells infected with adenovirus expressing GFP (AdGFP), but CUL3-deficient cells (AdCRE) failed to produce cGMP to comparable levels (Figure 8D). This suggests that loss of CUL3 in smooth muscle contributes to impaired $\mathrm{NO} / \mathrm{sGC} / \mathrm{cGMP}$ signaling through loss of sGC expression and cGMP production.

Given the association between impaired NO-mediated vasorelaxation and reduced guanylate cyclase activity in S-CUL3KO aorta, we tested the integrity of the cGMP-dependent pathway in promoting vasorelaxation. Aorta from S-CUL3KO mice exhibited reduced relaxation to the nonhydrolyzable cGMP 


\section{S-CUL3KO S-CUL3het Control}

A

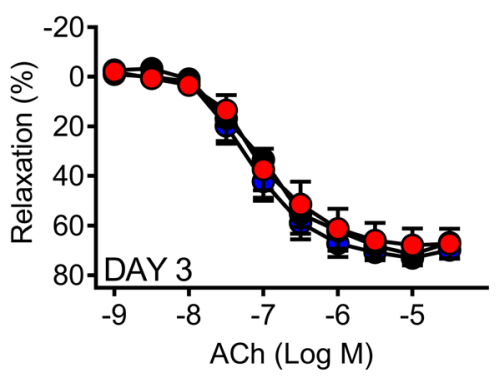

D

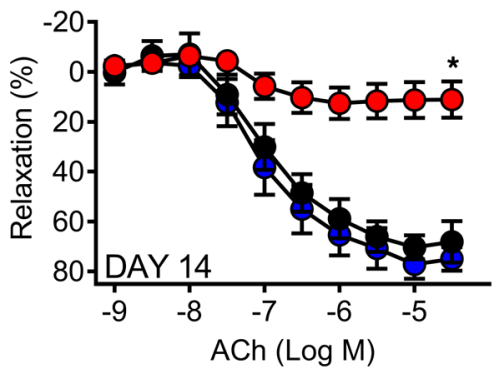

B

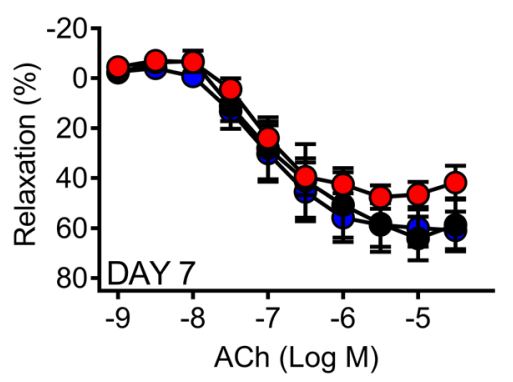

$\mathbf{E}$

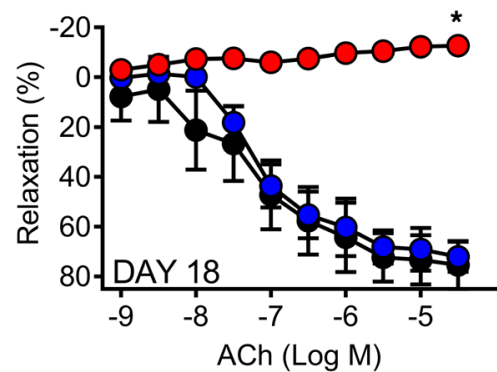

C

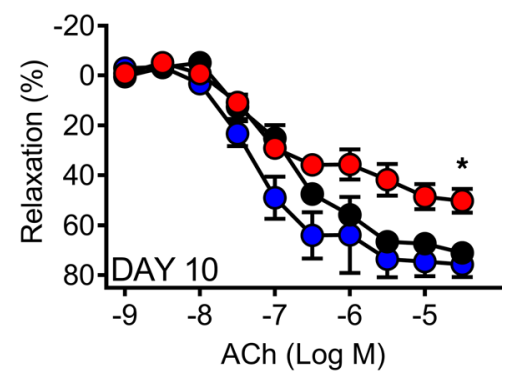

F

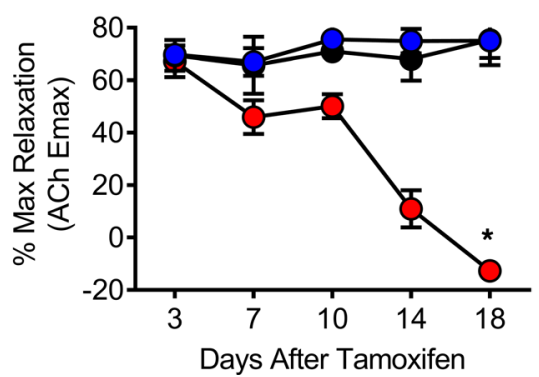

Figure 3. Time course of vasodilation. (A-E) Isometric tension was determined on aortic rings from the indicated mice. Dose-dependent relaxation to acetylcholine (Ach) was measured (following precontraction with prostaglandin $\mathrm{F}_{2 \alpha}\left[\mathrm{PGF}_{2 \alpha}\right]$ to $45 \%-50 \%$ of maximal PGF $\mathrm{F}_{2 \alpha}$ ) on days $3(\mathbf{A}, n=4-6$ / genotype), 7 (B, $n=4-6 /$ genotype), 10 (C, $n=4-6 /$ genotype), 14 ( $\mathbf{D}, n=4-7 /$ genotype), and 18 (E, $n=5-9 /$ genotype) following the last day of tamoxifen administration. (F) Percent maximal relaxation was also determined for all genotypes. For $\mathbf{A}-\mathbf{F}$, error bars represent mean $\pm \mathrm{SEM}$. ${ }^{*} P<0.05$ by 2-way (1-way in $\mathbf{F}$ ) repeated-measures ANOVA vs. other groups shown in each panel.

analog 8-pCPT-cGMP, as well as a heme-independent activator of sGC, BAY 58-2667 (Figure 9, A and B). This suggests that vessels from S-CUL3KO not only exhibit a deficit in cGMP production but also have an impaired response to cGMP. The Rho kinase inhibitor Y27632 did not correct the reduced relaxation in response to BAY 58-2667, suggesting that the poor response to cGMP is not dependent on contraction (Figure 9C). Vasodilation to the selective large conductance $\mathrm{Ca}^{2+}$-activated $\mathrm{K}^{+}$channel activator NS1619 was preserved, suggesting that the final common pathway required for vasodilation remains intact (Figure 9D).

To determine whether the impaired cGMP response is a direct result of CUL3 ablation, we examined PKG1 and Ser239 phosphorylation of vasodilator-stimulated phosphoprotein (VASP) in primary smooth muscle cells where CUL3 was either intact or ablated. PKG1 levels were modestly decreased in CUL3-deficient cells irrespective of the treatment with 8-pCPT-cGMP (Figure 9, E and F). VASP was robustly phosphorylated in aortic smooth muscle cells from control mice in response to cGMP. There was no significant difference in phosphorylated VASP at baseline between CUL3-deficient and control cells. Consistent with the decrease in PKG1, cGMP-induced phosphorylation of VASP was markedly impaired in aortic smooth muscle cells from S-CUL3KO mice. Together, these data suggest that the defect in NO-dependent relaxation results from both reduced sGC-dependent production of cGMP and reduced activity of cGMP in vascular smooth muscle.

\section{Discussion}

Hypertension is a complicated multifactorial disease encompassing numerous compensatory molecular and physiological pathways. Our studies implicate vascular smooth muscle CUL3 as an important regulator of $\mathrm{BP}$ and participant in the pathogenesis of hypertension. We were guided by several lines of evidence suggesting that CUL3 in smooth muscle cells could be important for regulating BP. First, mice expressing a dominant negative mutation (P467L) in the nuclear receptor transcription factor PPAR $\gamma$ selectively in vascular smooth muscle cell (S-P467L) exhibited a significant decrease in CUL3 protein expression in blood vessels, elevated levels of the CUL3 target RhoA, and elevated Rho kinase signaling, associated with impaired vascular function and hypertension $(11,13,35)$. Second, human subjects with dominant de novo 
A

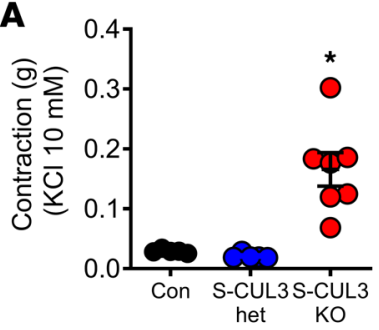

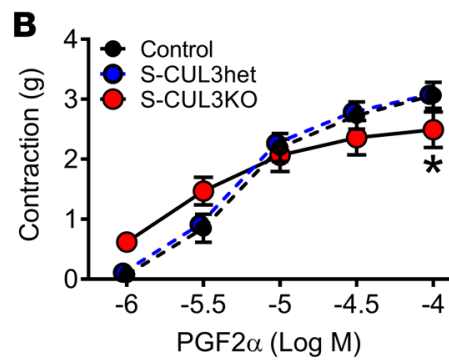

PGF2 $\alpha(\log M)$

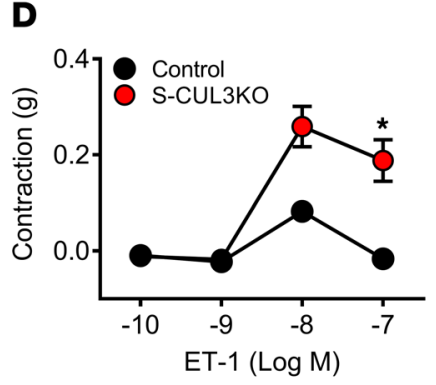

H

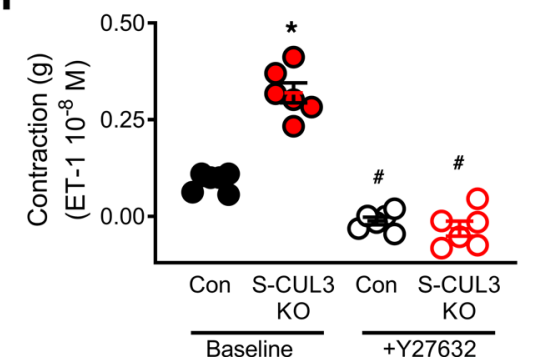

E

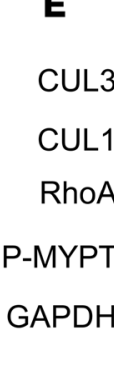

I

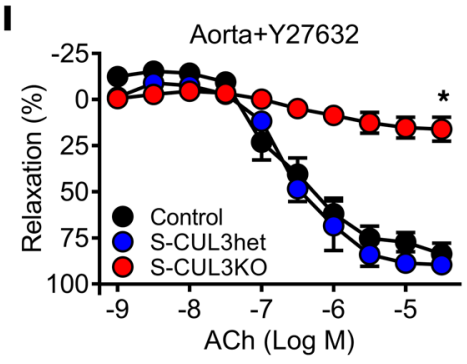

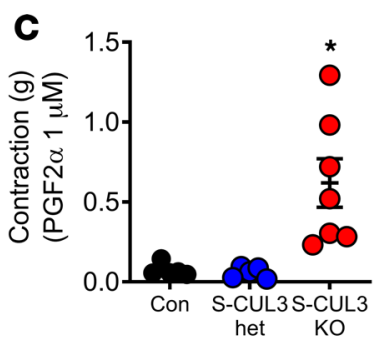

$\mathbf{F}$
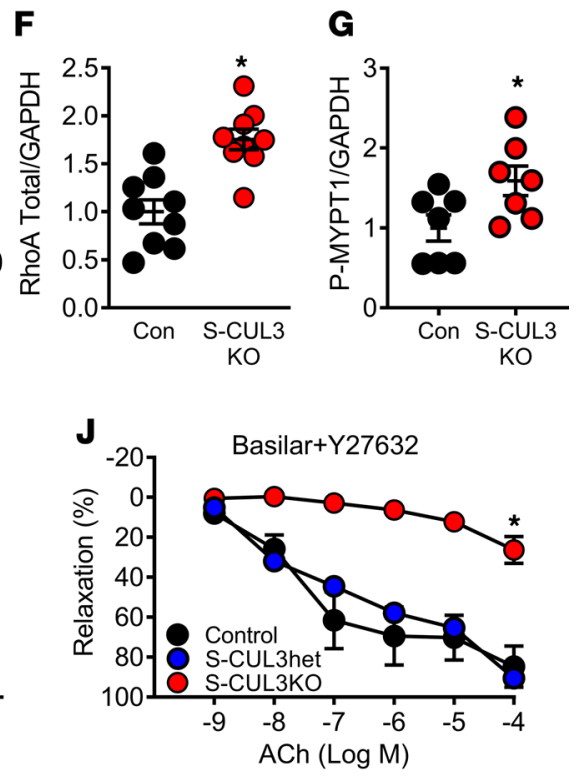

Figure 4. Vasocontraction of aorta: role of RhoA/ROCK. (A-D) Vasocontraction of thoracic aorta from the indicated mice (Con, control mice). Aorta rings were isolated and cleaned of perivascular adipose fat and were equilibrated for 45 minutes at $0.5 \mathrm{~g}$ tension; and vasocontraction responses to potassium chloride at $10 \mathrm{mmol} / \mathrm{L}$ (A, $n=5-7 /$ genotype), prostaglandin $\mathrm{F}_{20}\left(\mathrm{PGF}_{2 \alpha} ; \mathbf{B}, n=5-7 /\right.$ genotype), and endothelin 1 (D, ET-1, $n=4$ per genotype) were assessed. For $\mathbf{A}-\mathbf{D}$, error bars represent mean \pm SEM. ${ }^{*} P<0.05$ by 1-way (A and $\mathbf{C}$ ) or 2 -way repeated-measures ANOVA (B and $\left.\mathbf{D}\right)$. Note that the S-CUL3het graph in $\mathbf{B}$ was shifted 0.05 units in the $x$ and $y$ directions to clearly indicate the presence of 3 data sets on the graph because the control and S-CUL3 were essentially superimposed. Individual points for the $1 \mu \mathrm{M}$ dose are shown in C. (E) Western blot showing expression levels of indicated proteins in aorta from control and S-CUL3KO mice. Aortas were cleaned of perivascular fat and adventitia. Representative of 3 independent experiments. MYPT, myosin light chain phosphatase. (F and $\mathbf{G})$ Quantification of total RhoA (F) and P-MYPT1 (G) in aorta. For $\mathbf{F}$ and $\mathbf{G},{ }^{*} P<0.05$, S-CUL3KO vs. control by Student's $t$ test. (H) ET-1-induced vasoconstriction at $10 \mu \mathrm{mol} / \mathrm{L}$ in the absence or presence of the Rho kinase inhibitor $\mathrm{Y} 27632$ ( $n=6-8 / \mathrm{genotype}$ ). For $\mathbf{F}$ and $\mathbf{G}$, error bars represent mean $\pm \mathrm{SEM} .{ }^{*} P<0.05$ by 1 -way ANOVA. For $\mathbf{H},{ }^{*} P<0.05$ vs. control; ${ }^{*} P<0.05$ vs. baseline by one-way ANOVA. (I and J) Effect of the Rho kinase inhibitor Y27632 ( $1 \mu \mathrm{mol} / \mathrm{L}, 30$ minutes) on acetylcholine-mediated (Ach) relaxation in aorta (I, $n=4-6 /$ genotype) and basilar arteries (J, $n=5-7 /$ genotype). For I and J, error bars represent mean \pm SEM. ${ }^{*} P<0.05$ by 2 -way repeated-measures ANOVA.

CUL3 mutations exhibit severe early-onset hypertension (4). These mutations result in a form of CUL3 protein lacking 57 amino acids encoded by exon 9 (CUL3 39 ), which exhibits dominant negative activity $(13,36)$. Third, mice expressing the CUL $3 \Delta 9$ mutant protein either globally or selectively in smooth muscle are predisposed to arterial stiffness and mild hypertension $(15,16)$. The findings in this study are consistent with our hypothesis that smooth muscle CUL3 deficiency causes severe progressive hypertension and that defects associated with smooth muscle CUL3 function could contribute to the severity of disease in human patients. Indeed, evidence suggests that vascular smooth muscle CUL3 is at least as physiologically important to BP regulation as CUL3 expressed in the epithelial cells of the kidney $(14,17,37)$.

Impairment of vascular function is involved in the pathogenesis of human hypertension (38). The endothelium produces NO from L-arginine, which diffuses through the intracellular space to induce vasorelaxation of smooth muscle. eNOS-deficient mice are severely hypertensive, demonstrating that endothelium-derived NO is a key vasoactive agent $(39,40)$. Expression of both total eNOS and phosphorylated eNOS (active form) was not altered in S-CUL3KO mice, suggesting that NO production is unlikely to be impaired. Endogenous NO bioavailability may be mildly decreased due to oxidative stress, as evidenced by increased Nox-2, HO-1, and nitrotyrosine staining. However, the diminished vasorelaxation is unlikely 
A
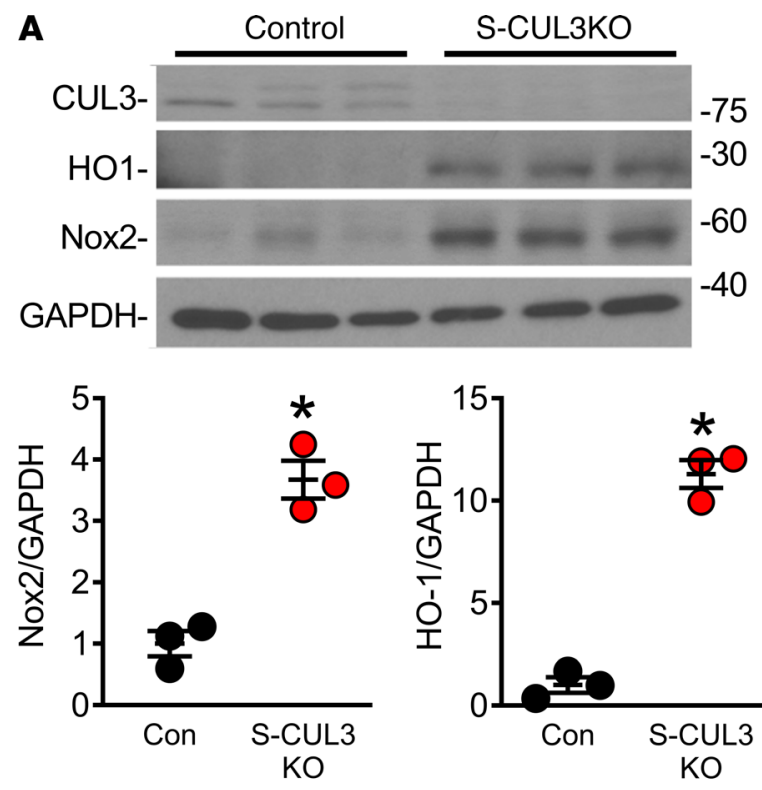

C

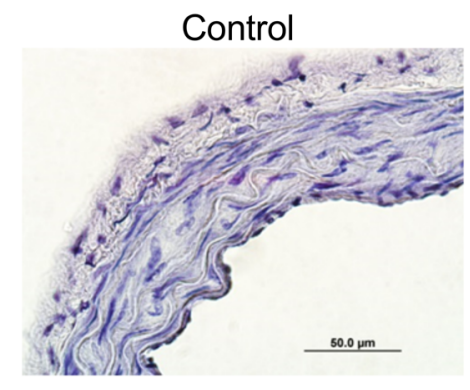

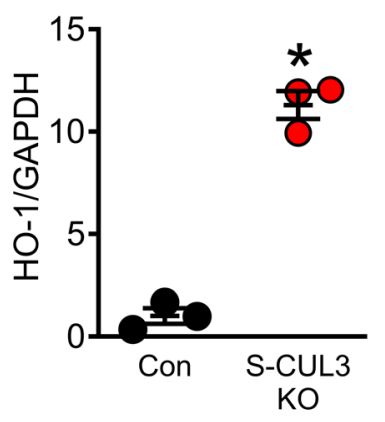

S-CUL3KO

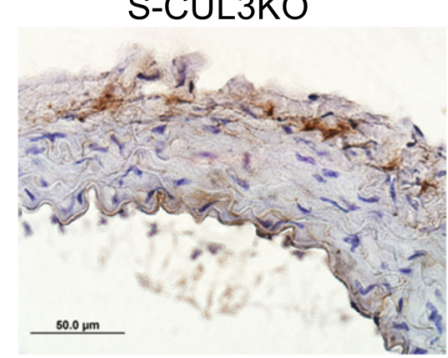

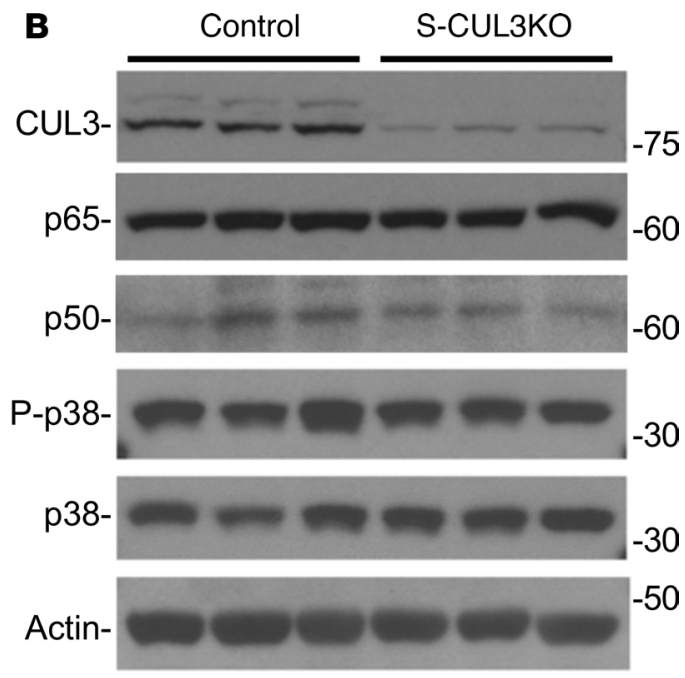

D
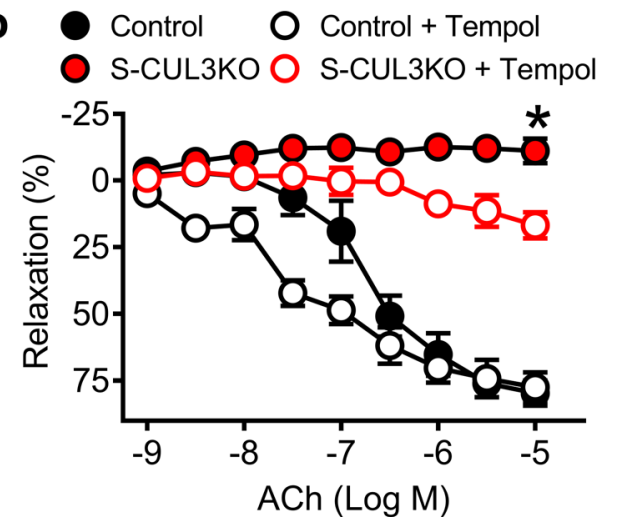

Figure 5. Oxidative stress. (A) Western blot showing expression levels of the indicated proteins in aorta. Bottom panels show quantification of heme oxygenase 1 (H01) and gp91phox (Nox2) protein. For A, error bars represent mean \pm SEM. ${ }^{*} P<0.05$, S-CUL3KO vs. control (Con) by Student's $t$ test. (B) Western blot showing expression levels of the indicated proteins in aorta cleaned of adventitia and perivascular adipose tissue. (C) Cross sections of aorta showing immunohistochemistry for nitrotyrosine (brown staining). Scale bars: $50 \mu \mathrm{m}$. (D) Dose-dependent relaxation to acetylcholine (Ach) in an independent cohort of aorta from the indicated mice in the absence and presence of the superoxide scavenger Tempol ( $1 \mathrm{mmol} / \mathrm{L}, 30-\mathrm{minute}$ preincubation, $n=4-6$ / genotype). For $\mathbf{D}$, error bars represent mean \pm SEM. ${ }^{*} P<0.05$, S-CUL3KO vs. the other groups by 2 -way repeated-measures ANOVA.

a direct result of decreased NO bioavailability, because even supraphysiological doses of exogenous NO fail to correct the impairment of vasorelaxation in S-CUL3KO mice. Thus, our results strongly suggest that decreased smooth muscle responsiveness to NO, likely occurring at the level of the NO receptor SGC, is the immediate cause of impaired vasorelaxation. Consistent with this, expression of both subunits of sGC, sGC $\alpha 1$ and sGC $\beta 1$, were significantly downregulated at the mRNA and protein levels; and this downregulation was associated with reduced SNP-induced cGMP production. This is in agreement with evidence that sGC $\alpha 1$ - or sGC $\beta 1$-knockout mice are hypertensive (41-43). Moreover, the loss of vasorelaxation could contribute to increased responses to $\mathrm{G}$ protein-coupled receptor agonists such as ET-1, 5-HT, and PGF ${ }_{2 a^{\circ}}$. Although, loss of sGC $\alpha 1$ and sGC $\beta 1$ is likely to be responsible for the loss of NO responsiveness observed in S-CUL3KO mice, they are unlikely to be targets of CUL3. This is because a loss of CUL3, which normally stimulates ubiquitination and proteasomal degradation of its target substrates, would be expected to result in a marked increase in a target protein, as it does for RhoA (11). Moreover, that the decrease in sGC $\alpha 1$ and sGC $\beta 1$ proteins was associated with a marked decrease in their mRNAs suggests a transcriptional or posttranscriptional response. However, there was no change in the expression of 2 proteins (HuR1 and AUF1) that have been previously reported to regulate stability of sGC $\alpha 1$ and sGC 1 mRNAs (30-33). Thus, how CUL3 regulates the expression of the subunits of SGC remains to be determined. It will be interesting to assess whether CUL3 regulates the levels of the Forkhead box subclass O transcription factors, 


\section{S-CUL3KO \\ S-CUL3het \\ Control}
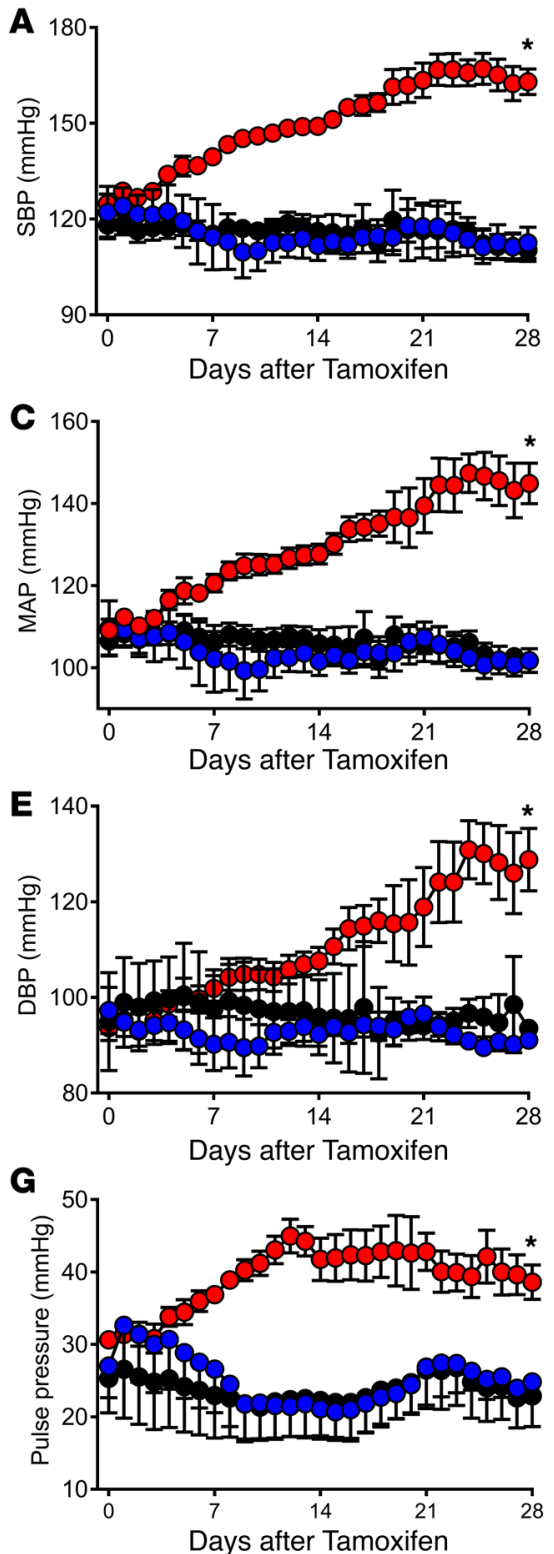
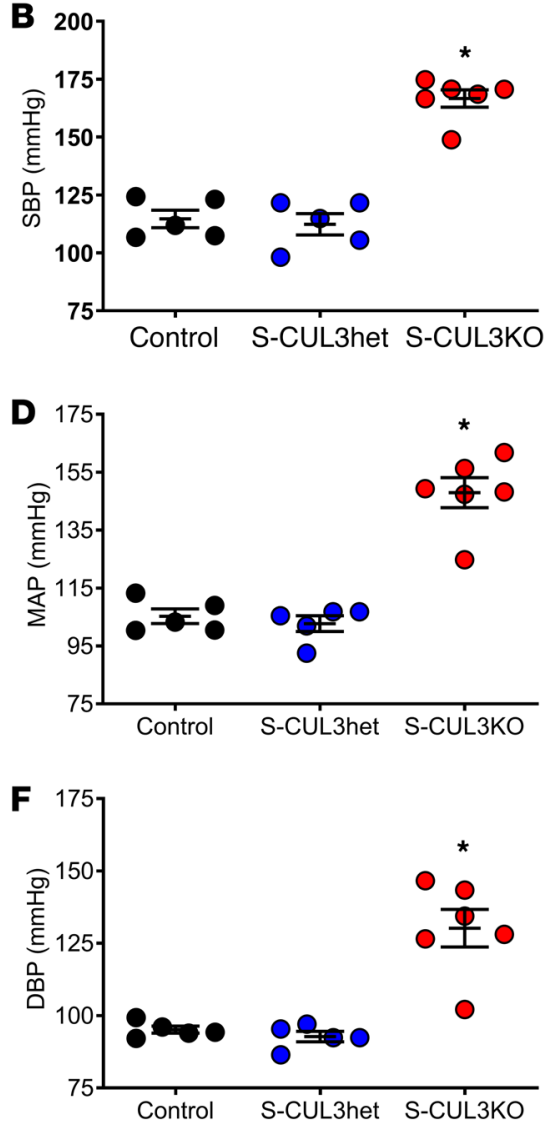

H

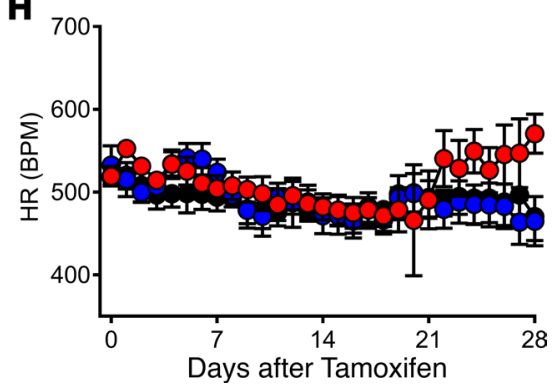

Figure 6. Hypertension in S-CUL3KO mice. BP was measured continuously and recorded every 5 minutes for 10 seconds by radiotelemetry. Systolic BP (A; SBP), mean arterial pressure (C; MAP), and diastolic BP (E; DBP) measurements represent daily 24-hour averages over 1 month following the last day of tamoxifen administration (day 0$)(n=$ 5-6/genotype). Summary SBP (B), MAP (D), and DBP (F) during the fourth week after tamoxifen administration. Data represent 24 -hour averages over 7 days of measurements. Pulse pressure (C), and heart rate $(\mathbf{H} ; \mathrm{HR})$ measurements represent daily 24-hour averages. For $\mathbf{A}-\mathbf{H}$, error bars represent mean \pm SEM. ${ }^{*} P<0.05$ S-CUL3KO vs. control groups by 2-way repeated-measures ANOVA (A, C, $\mathbf{E}$, and $\mathbf{G}$ ) or 1-way ANOVA (B, D, and F).

as they were recently demonstrated to regulate transcription of both sGC $\alpha$ and sGC $\beta$ (44). This highlights the need to identify novel CUL3 substrates in the vascular smooth muscle and connect these targets with identified CUL3-sensitive mediators of $\mathrm{NO} / \mathrm{sGC} / \mathrm{cGMP}$ signaling.

$\mathrm{NO}$ is the key inducer of sGC-mediated production of cGMP. We provide evidence that cGMP production is decreased in CUL3-deficient smooth muscle cells. This is consistent with published reports showing that cGMP production is impaired following loss of sGC expression $(28,43,45)$. Another interpretation of these data could be that cGMP is produced but is rapidly degraded by PDE5, the main PDE involved in cGMP degradation in vascular smooth muscle cells. Indeed, we recently identified PDE5 as a CUL3 target (22). However, PDE5 expression was not increased but rather decreased in aorta of S-CUL3KO mice (data not shown). Additionally, aorta from S-CUL3KO mice showed impaired relaxation to a nonhydrolyzable cGMP analog (8-pCPT-cGMP), indicating that preventing PDE-mediated degradation did not restore normal relaxation responses in the absence of CUL3. It is possible that longer incubation with 8-pCPT-cGMP would lead to increased relaxation. This is based on the observation that higher concentrations of 8-pCPT-cGMP caused increased relaxation compared with lower doses, where relaxation was markedly impaired. The decrease in cGMP action was likely a consequence of a decrease in PKG1 expression in 


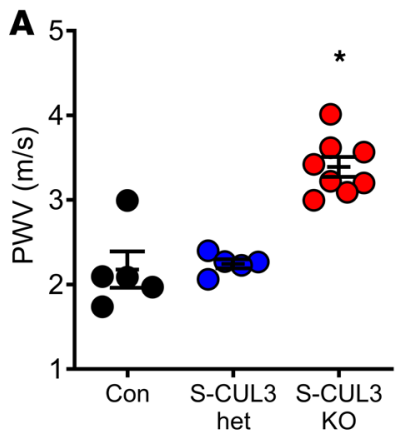

D
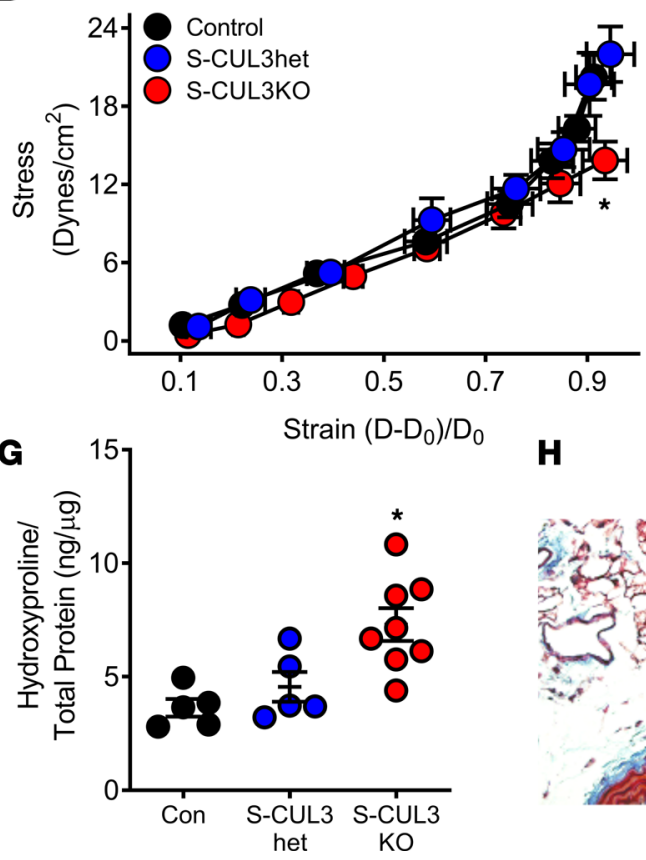

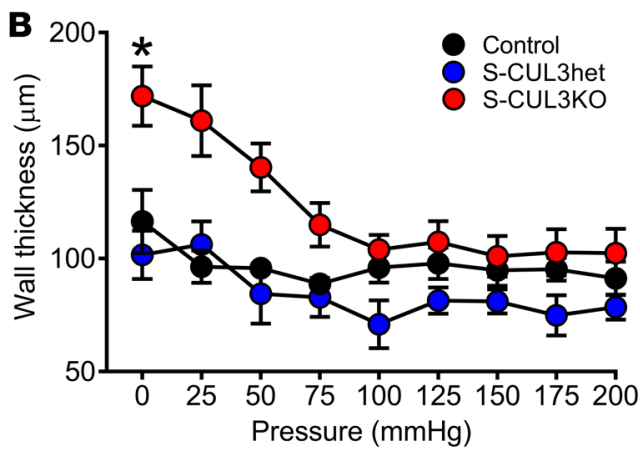

E

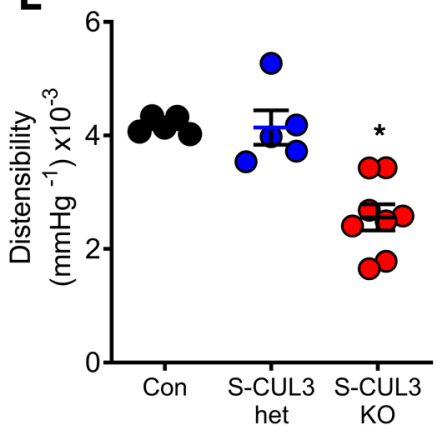

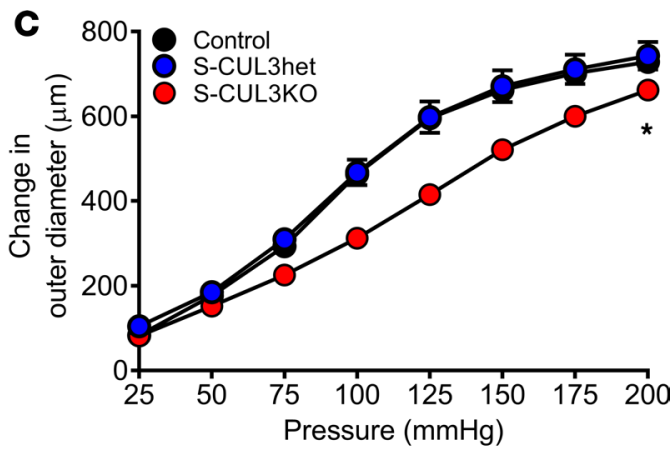

$\mathbf{F}$

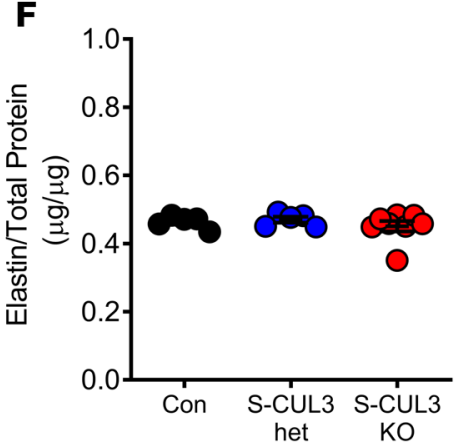

Figure 7. Arterial stiffness. (A) Pulse wave velocity (PWV) measurements were determined 4 weeks after tamoxifen administration by Doppler ultrasound. $n=7-16 /$ genotype; ${ }^{*} P<0.05$ S-CUL3KO vs. controls (Con) by 1-way ANOVA. (B-D) Wall thickness (B, $n=5-8 /$ genotype), pressure-diameter curves (C, $n=5-8$ /genotype), and stress-strain relationships ( $\mathbf{D}, n=5-8$ /genotype). In $\mathbf{D}$, $\mathbf{D}$ denotes the outer diameter at one of pressures studied, while $\mathrm{D}_{0}$ stands for the outer diameter at baseline. ${ }^{*} P<0.05$ calculated by 2 -way ANOVA. (E-G) Aortic distensibility (E), elastin (F), and aortic collagen content as measured by hydroxyproline assay $(\mathbf{G}) .{ }^{*} P<0.05$ calculated by 1 -way ANOVA. For A-G, error bars represent mean $\pm \mathrm{SEM}$. (H) Adventitial collagen was determined by Masson's trichrome staining. Original magnification, $\times 40$. Scale bars: $100 \mu \mathrm{m}$.

smooth cells from S-CUL3KO mice. Indeed, ablation of CUL3 in primary aortic smooth muscle cells was associated with a decrease in PKG1 protein and impaired phosphorylation of VASP at Ser239. As PKG activity can also be regulated by its oxidation state, it may be interesting in future studies to assess whether CUL3 regulates the oxidation state of PKG (46). Further downstream, vasorelaxation to the BK channel activator NS1619 was comparable in control and S-CUL3KO aortas, indicating that the downstream pathway promoting relaxation remains intact in the absence of CUL3. These observations suggest that the defect in the CUL3/NO/sGC/cCMP pathway occurs at multiple levels - sGC expression, production of cGMP, and activity of cGMP in vascular smooth muscle.

We showed previously that mice selectively expressing a dominant negative PPAR $\gamma$ mutation in smooth muscle (S-P467L) exhibited modest hypertension that was recapitulated in mice expressing the CUL3 mutation selectively in smooth muscle (S-CUL3 $\Delta 9)(10,11,16)$. In contrast, S-CUL3KO mice exhibited some of the highest BPs observed in mice. The reason for the heightened BP response could be multifactorial. First, RhoA is a bona fide substrate of CUL3 in vascular smooth muscle (13). The RhoA/Rho kinase pathway was elevated in aorta from S-CUL3KO mice and was required for enhanced contraction, suggesting an increase in basal vascular tone, which could contribute to increase 
A
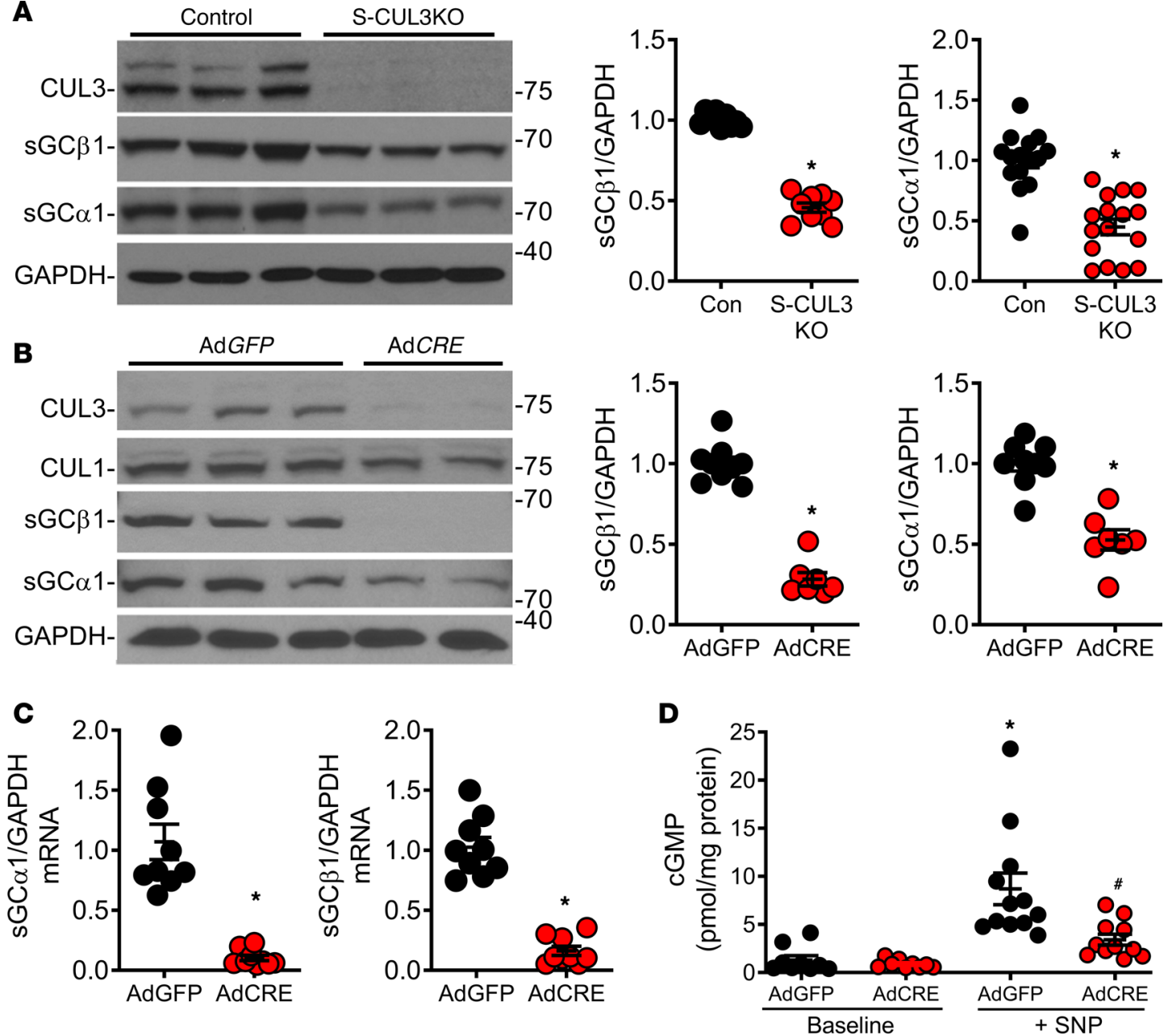

Figure 8. Regulation of cGMP production. (A) Representative Western blot of indicated proteins in aorta (cleaned of adventitia and perivascular adipose tissue) with quantification of the soluble guanylate cyclase subunits sGC $\beta 1$ and $s G C \alpha 1 . n=9-16 /$ genotype; ${ }^{*}<<0.05$ vs. control (Con) by Student's $t$ test. (B) Primary mouse aortic smooth muscle cells were isolated from CUL $3^{f / f l}$ mouse aorta and infected with AdCRE or AdGFP for 72 hours. Expression of the indicated proteins and quantification is shown. $n=7-9 /$ genotype. Representative of three independent experiments. ${ }^{*} P<0.05$ vs. AdGFP by Student's $t$ test. (C) Expression of sGC $\beta 1$ and sGC $\alpha 1$ mRNAs in smooth muscle cells 72 hours after AdCRE or AdGFP infection. $n=9 /$ genotype; ${ }^{*} P<0.05$ vs. AdGFP. (D) cGMP content was measured in primary aortic smooth muscle cells infected with AdCRE or AdGFP, and subsequently exposed to vehicle (baseline) or to sodium nitroprusside (SNP; $10 \mu \mathrm{mol} / \mathrm{L}$ ) in the presence of the pan-phosphodiesterase inhibitor 3-isobutyl-1-methylxanthine (IBMX; $100 \mu \mathrm{mol} / \mathrm{L}) . n=9-12$ / genotype/treatment from 3 independent experiments. ${ }^{*} P<0.05$ vs. all groups, ${ }^{*} P<0.05$ vs. baseline. For $\mathbf{A}-\mathbf{D}$, error bars represent mean $\pm S E M$.

in vascular peripheral resistance. Second, endothelium-dependent and -independent relaxation responses were severely abrogated in S-CUL3KO aorta, suggesting that a loss of NO responsiveness could be a major contributor to the BP phenotype. It is notable that Rho kinase inhibition did not restore normal responsiveness of S-CUL3KO aorta to NO-mediated vasorelaxation, suggesting that CUL3 affects contraction and relaxation pathways independently. Third, nitrotyrosine, HO-1, and NOX2 - all markers of oxidative stress - were elevated in aorta of S-CUL3KO mice. Although there was evidence of oxidative stress, scavenging superoxide only modestly improved vascular dysfunction, suggesting that an alternative pathway may be more predominant. Fourth, a reduction in sGC expression directly contributed to reduced cGMP production and impairment of the protective vasodilatory mechanism. We proposed that this could be the dominant mechanism that contributes to the severity of hypertension in S-CUL3KO mice. Last, another contributing factor to the severe hypertension in the S-CUL3KO mice was the increase in thickness of the aortic tunica media. An increase in medial wall thickness or smooth muscle cell proliferation has been shown to be correlated with the pathogenesis of hypertension $(47,48)$.

Arterial stiffening is associated with cardiovascular events such as heart failure, renal failure, and stroke, and has been shown to be an independent predictor of end organ damage in hypertensive subjects (49). 


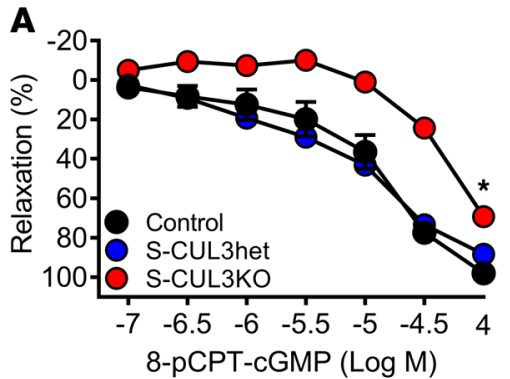

C

Control O Control + Y 27632

O S-CUL3KO O S-CUL3KO + Y27632

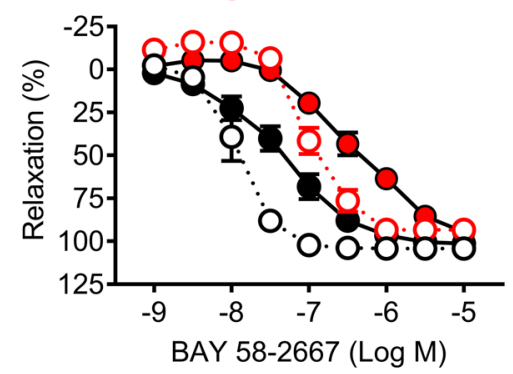

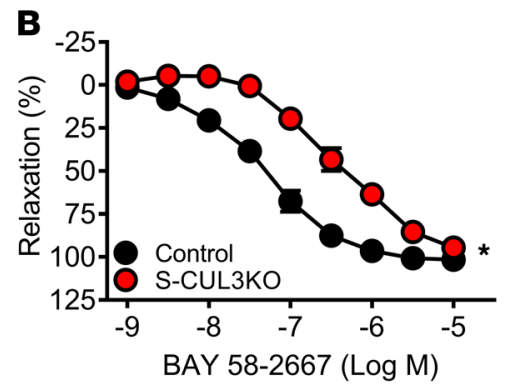

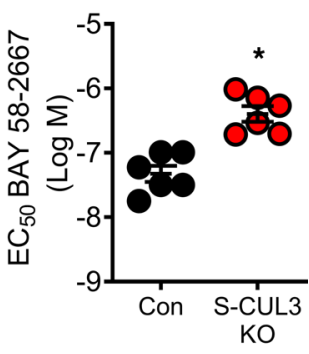

D

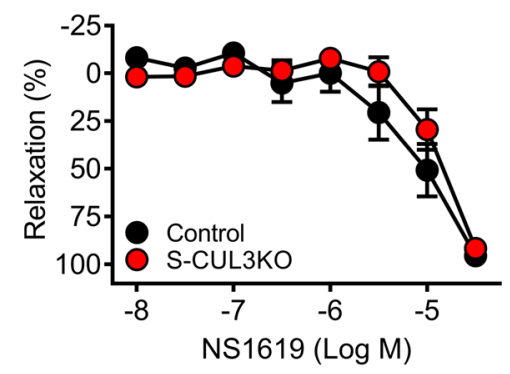

F

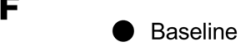

8-cCPT-cGMP
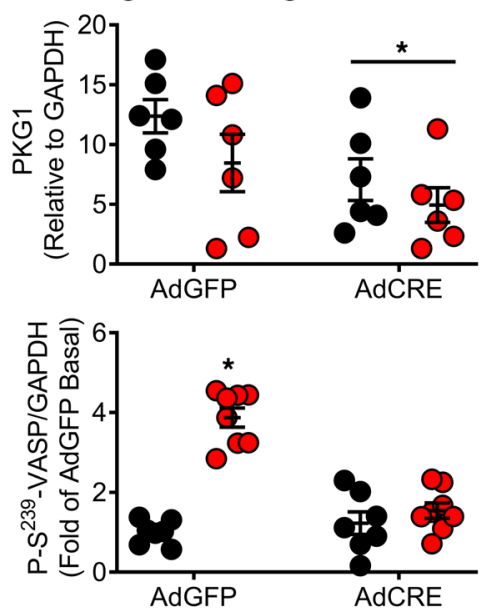

PKG1-

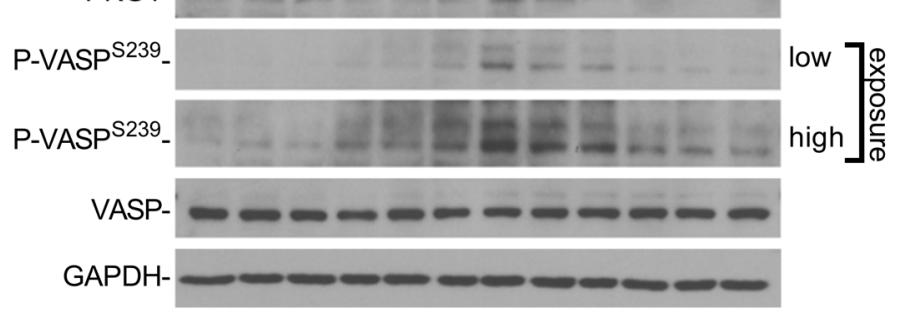

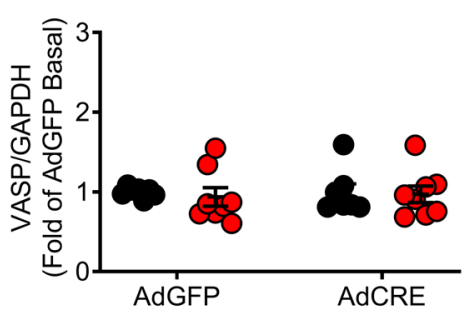

Figure 9. Vasodilation to NO/sGC/cGMP pathway intermediates. (A and B) Relaxation of aortic rings was measured in response to the nonhydrolyzable cGMP analog 8-pCPT-cGMP (A) or the sGC activator BAY 58-2667 (cinaciguat) (B). Dose-dependent relaxation curves (left) and quantification of maximal relaxation (right) are shown ( $n=4-6 /$ genotype). (C) Dose-dependent relaxation of aorta to BAY 58-2667 in the absence and presence of the Rho kinase inhibitor Y27632 (1 $\mu \mathrm{mol} / \mathrm{L}, 30$ minutes, $n=5$-6/genotype). (D) Dose-dependent relaxation of aorta to the BK channel activator NS1619 ( $n=6 / g e n o t y p e)$. For A-D, error bars represent mean \pm SEM. ${ }^{*} P<0.05$ vs. controls (Con) by 1- or 2-way repeated-measures ANOVA. (E) Aortic smooth muscle cells isolated from CUL $3^{f / f l}$ mice were grown in vitro and infected with AdGFP or AdCRE. Cells were then incubated with 8-pCPT-cGMP or DMSO control for 15 minutes and lysates collected. Western blot showing levels of the indicated proteins in CUL3-deficient primary aortic smooth muscle cells treated with 8-pCPT-cGMP (100 $\mu \mathrm{mol} / \mathrm{L}, 15$ minutes). The gel is representative of 3 independent experiments. (F) The level of PKG1, P-VASP5239, and total VASP was quantified ( $n=$ 7 -8/treatment). For $\mathbf{F}$, error bars represent mean \pm SEM. ${ }^{*} P<0.05$ by 2 -way ANOVA. VASP, vasodilator-stimulated phosphoprotein. For PKG1, significance was observed when comparing the effect of AdCRE but not the effect of cGMP.

We measured PWV as an index of aortic stiffness in S-CUL3KO mice. Consistent with published results showing that eNOS-knockout mice, a model for NO resistance, exhibit increased PWV, S-CUL3KO mice showed elevated PWV and reduced aortic distensibility (50). This is in agreement with our data showing severe loss of NO responsiveness and increased adventitial collagen deposition. These findings recapitulate reports showing that PWV is increased in mice expressing CUL $3 \Delta 9$ both globally and selectively in the vascular smooth muscle, and further validate that CUL3 dysfunction in the smooth muscle is an independent contributor to arterial stiffness $(15,16)$.

As mentioned above, CUL3 mutations were first identified in FHHt patients who exhibited electrolyte abnormalities consistent with a defect in the kidney (4). Our studies provide evidence of a critical role for smooth muscle CUL3 in BP regulation. However, we recognize that our studies cannot rule out the possible contribution of the renal vasculature as a contributor to hypertension in S-CUL3KO mice. 
A decrease in renal NO bioavailability has been shown to result in increased renal vasoconstriction, decreased renal blood flow, and hypertension via upregulation of angiotensin II production (51). However, circulating angiotensin II production was not increased in plasma of S-CUL3KO mice. Future studies will focus on the role of the kidney and any changes in renal vascular resistance. Our studies were conducted in aorta, a conduit blood vessel, and were replicated in basilar artery, a cerebral resistance vessel. Nevertheless, we recognize that vascular resistance controlling BP is largely determined by the contractile state of peripheral resistance arteries, and thus further studies in peripheral resistance vessels are warranted.

Additional support for examining a renal mechanism is derived from evidence that WNKs are targets of CUL3 in the kidney and are expressed in smooth muscle cells in addition to sodium-transporting epithelial cells. WNK3 has been shown to potentiate the effects of angiotensin II in the aorta through phosphorylation of NKCC1 in the smooth muscle cell, an effect that is impaired in WNK3-deficient mice (52). Similarly, mice heterozygous for $W N K 1$ exhibit decreased aortic smooth muscle contractility and a decreased myogenic response, suggesting that WNK1 is a contributor to basal BP (53). WNK4 depletion has been shown to increase vasoconstriction through TRPC3-induced calcium influx in smooth muscle cells (54). Thus, there is a plausible rationale for assessing the importance of WNK kinases as targets of CUL3 in the S-CUL3KO model.

In conclusion, our study provides compelling evidence for the sufficiency of vascular smooth muscle CUL3 as a regulator of BP. The magnitude of hypertension and NO impairment with smooth musclespecific CUL3 ablation was much greater than with smooth muscle-specific expression of a dominant negative mutation in CUL3 (CUL3 $\Delta 9$ ), which is sufficient to cause hypertension in humans $(4,16)$. It is likely that preservation of some fraction of CUL3 activity in S-CUL3 $\Delta 9$ mice is sufficient to restrain the phenotype and that the complete ablation of CUL3 markedly alters the proteome. Thus, future proteomics studies will be needed to identify CUL3-interacting partners and substrates in smooth muscle and other cardiovascular tissues to better understand the role of CUL3 in BP regulation.

\section{Methods}

Animals. Mice carrying a conditional allele of CUL3 (CUL3 $3^{\text {flff }}$ ) (10 weeks old; backcrossed to C57BL/6J mice at least 10 generations) were a gift from Jeffrey Singer, Department of Biology, Portland State University. $C U L 3^{f / f l}$ mice were bred with mice expressing a tamoxifen-inducible Cre recombinase $\left(C_{\text {reERT }}^{2}\right)$ under the control of the smooth muscle myosin heavy chain promoter (B6.FVB-Tg [Myh11-cre/ERT2]1Soff/J; The Jackson Laboratory, stock 019079) for 2 successive generations. $\mathrm{Cre}^{+} / C U L 3^{f l f l}$ or $\mathrm{Cre}^{+} / C U L 3^{f /+}$ mice were treated with tamoxifen $(75 \mathrm{mg} / \mathrm{kg})$ i.p. daily for 5 consecutive days to generate S-CUL3KO or heterozygous mice. $\mathrm{Cre}^{+} / C U L 3^{f / f l}$ mice were simultaneously administered corn oil as controls. Because the Myh11-cre/ERT2 transgene was inserted on the Y chromosome, only male mice could be used in this study. Age-matched S-CUL3KO, S-CUL3het, and littermate control mice were used in this study. CUL3 $3^{\text {fl/fl }}$ mice were maintained on a C57BL/6 genetic background.

Cell culture. Primary smooth muscle cells were isolated from thoracic aorta of $C U L 3^{\text {fl/fl}}$ mice and validated as previously described (55). Briefly, aortas were dissected and cleaned of perivascular adipose tissue and cut into approximately 1- to 2-mm pieces. Aorta pieces were digested with collagenase type II for 6 hours, centrifuged, and plated in a 48 -well plate. Cells were allowed to grow to confluency, passaged, and characterized as smooth muscle by staining with $\alpha$-smooth muscle actin. Primary smooth muscle cells were maintained in high-glucose DMEM supplemented with 10\% FBS, $1 \%$ glutamine, and 1\% penicillin and streptomycin at $37^{\circ} \mathrm{C}$ in a $5 \% \mathrm{CO}_{2}$ incubator. The cultured primary smooth muscle cells from $\mathrm{Cre}^{+} / \mathrm{CUL} 3^{\text {fl/f }}$ mice were infected with AdCRE to express Cre recombinase and generate smooth muscle-deficient CUL3 cells. $\mathrm{Cre}^{+} / C U L 3^{\text {fl/fl}}$ primary smooth muscle cells were infected with AdGFP as controls. Cells were collected in TRIzol reagent (Thermo Fisher Scientific) and stored at $-80^{\circ} \mathrm{C}$ for quantitative PCR analysis.

cGMP and angiotensin peptide measurement. Cells were also lysed in RIPA buffer (1\% Nonidet P-40, 0.5\% deoxycholate, and $0.1 \%$ SDS in $1 \times$ PBS with proteinase inhibitors; Roche). Total protein lysates were collected and stored at $-80^{\circ} \mathrm{C}$ until further analysis. Aortas from control and S-CUL3KO mice were cleaned of adventitia fat, frozen in liquid nitrogen, and homogenized in RIPA buffer containing protease inhibitors. Total protein lysates were collected and stored at $-80^{\circ} \mathrm{C}$ until further analysis. Equal amounts of protein lysates were separated by SDS-PAGE and transferred onto a nitrocellulose membrane (GE Healthcare). Membranes were blocked with $5 \%$ milk or BSA and incubated at $4{ }^{\circ} \mathrm{C}$ overnight with primary antibodies. The sources of antisera were as follows: Cullin-3 (catalog A301-109A, Bethyl Laboratories), Cullin-1 (catalog A303-373A, Bethyl Laboratories), Cullin 5 (catalog A302-173A, Bethyl Laboratories), GAPDH (catalog sc-32233, Santa Cruz 
Biotechnology Inc.), RhoA (catalog 2117, Cell Signaling Technology), HO-1 (catalog 70081, Cell Signaling Technology), phospho-VASP (catalog 3114, Cell Signaling Technology), VASP (catalog 3132, Cell Signaling Technology), eNOS (catalog 9572, Cell Signaling Technology), phospho-eNOS (Ser1177) (catalog 9571, Cell Signaling Technology), PKG1 (catalog 3248, Cell Signaling Technology), phospho-MYPT1 (catalog 5163, Cell Signaling Technology), p65 (catalog 4764, Cell Signaling Technology), p38 MAPK (catalog 9212, Cell Signaling Technology), phospho-p38 (catalog 9212, Cell Signaling Technology), gp91 ${ }^{\text {phox }}$ (catalog 553370, BD Biosciences), sGC $\beta 1$ (catalog 154841, Abcam), sGC $\beta 1$ (catalog 160897, Cayman Chemical), AUF1 (catalog ab50692, Abcam), sGC 1 1 (catalog ab50385, Abcam), $\beta$-actin (catalog ab16039, Abcam), CYB5R3 (catalog 10894-1-AP, Proteintech), HuR (catalog 1910-1-AP, Proteintech). Immunoblots were incubated with a secondary antibody conjugated to horse radish peroxidase and developed using ECL (Amersham Biosciences). Protein bands were quantified using ImageJ software (NIH).

cGMP was quantified by measuring the production of cGMP in CUL3-deficient primary aortic smooth muscle cells with a standard cGMP ELISA assay kit (catalog 581021, Cayman Chemical). $\mathrm{Cre}^{+} / C U L 3^{f / f l}$ primary smooth muscle cells were infected with AdCRE or AdGFP as controls. Three days after infection, cells were incubated with serum-free media solution containing $10 \mu \mathrm{M}$ 3-isobutyl-1-methylxanthine (IBMX) and $10 \mu \mathrm{M}$ SNP for 20 minutes at $37^{\circ} \mathrm{C}$. Cells were lysed using a buffer provided by the manufacturer, and total protein concentrations were quantified by Lowry assay with BSA as standard using a DC protein assay kit (catalog 5000111, Bio-Rad). Both basal and SNP-stimulated cells lysates were run in triplicate using a cGMP standard provided by the manufacturer, and the results are expressed as picomoles cGMP per milligram of total protein.

The concentration of angiotensin peptides in the plasma was measured using commercially available ELISAs (RayBiotech) as we reported (56).

Quantitative PCR. Total RNA was extracted from thoracic aorta or from primary aortic smooth muscle cells using the TRIzol phenol chloroform extraction method (Invitrogen) and an RNA Mini Kit (Invitrogen). RNA quality and concentrations were determined using a NanoDrop analyzer. Reverse transcription was conducted using SuperScript III (Invitrogen) to generate cDNA, and the cDNA samples were stored at $-80^{\circ} \mathrm{C}$ until further use. Quantitative PCR analysis was determined by TaqMan gene expression assays (Applied Biosystems) from $10 \mathrm{ng}$ cDNA in a total volume of $10 \mu \mathrm{L}$ according to the manufacturer's instructions. The assay numbers for all TaqMan probes used are as follows: Mn99999915_g1 (mouse GAPDH), Mm00516926_m1 (mouse sGCß1), Mm01220285_m1 (mouse sGC $\alpha 1$ ), Mm00516011_m1 (mouse HuR), Mm01201314_m1 (mouse AUF1), Mm01336510_m1 (mouse CUL3), Mm00504077_m1 (mouse CYB5R3). All results were normalized to housekeeping gene GAPDH and quantified by the $\triangle \triangle \mathrm{CT}$ method (57).

$B P$ measurements. BP was measured by radiotelemetry as previously described (16). Briefly, mice were implanted with radiotelemeter transmitters through the left carotid artery and allowed to recover for 7 days. Three-month-old $\mathrm{Cre}^{+} / C U L 3^{f / f l}$ or $\mathrm{Cre}^{+} / C U L 3^{f /+}$ mice were anesthetized with ketamine $(87.5 \mathrm{mg})$ and xylazine $(12.5 \mathrm{mg} / \mathrm{kg})$, the catheter was placed into the left carotid artery, and the transmitter was subcutaneously placed along the left flank. Mice were allowed to recover for 7 days, after which SBP, MAP, DBP, heart rate, and activity were recorded every 5 minutes for 10-second intervals for a total of 7 days. $\mathrm{Cre}^{+} / C U L 3^{f / / f l}$ or $\mathrm{Cre}^{+} / C U L 3^{f /+}$ mice were then treated with tamoxifen $(75 \mathrm{mg} / \mathrm{kg}$ i.p.) or corn oil for 5 consecutive days, and BP was measured continuously for a total duration of 4 weeks. Baseline BP was recorded every 5 minutes for 10 seconds, and radiotelemeters were turned off. After the last day of tamoxifen i.p. injection, radiotelemeters were turned back on, and BP was recorded continuously every 5 minutes for 10 seconds for 1 month. All data were initially collected and stored using Dataquest ART and then on an upgraded radiotelemetry platform using the Ponemah software package (DSI).

Wire and pressurized myograph studies. Briefly, thoracic aortas were isolated and cleaned of adventitia fat, and vascular reactivity experiments were conducted in a suspended organ bath using wire myograph as previously described (11). Mice were euthanized with a lethal dose of Euthasol (150 mg/kg i.p), and the thoracic aorta was dissected free of adventitia fat, cut into rings 3-4 mm in length, and suspended in an organ bath containing Krebs buffer $\left(118.3 \mathrm{mmol} / \mathrm{L} \mathrm{NaCl}, 4.7 \mathrm{mmol} / \mathrm{L} \mathrm{KCl}, 2.5 \mathrm{mmol} / \mathrm{L} \mathrm{CaCl}_{2}, 1.2\right.$ $\mathrm{mmol} / \mathrm{L} \mathrm{MgSO}_{4}, 1.2 \mathrm{mmol} / \mathrm{L} \mathrm{KH}_{2} \mathrm{PO}_{4}, 25 \mathrm{mmol} / \mathrm{L} \mathrm{NaHCO}_{3}, 11 \mathrm{mmol} / \mathrm{L}$ glucose) bubbled with 95\% $\mathrm{O}_{2} / 5 \% \mathrm{CO}_{2}$, maintained at $37^{\circ} \mathrm{C}$. Aortic rings were connected to a force transducer to measure isometric tension, and following equilibration at $0.5 \mathrm{~g}$ for 45 minutes, precontracted with $\mathrm{PGF}_{2 \alpha}$ to $45 \%-50 \%$ of maximal response to U46619. After a plateau was reached, concentration-response curves were determined for ACh (0.001-100 $\mu \mathrm{mol} / \mathrm{L})$, methacholine $(0.01-100 \mu \mathrm{mol} / \mathrm{L}), \mathrm{SNP}(0.001-100 \mu \mathrm{mol} / \mathrm{L})$, DEA NONOate 
(0.001-100 $\mu \mathrm{mol} / \mathrm{L}), 8$-(4-chlorophenylthio)cyclic-3',5'-GMP (8-pCPT-cGMP) (0.1-100 $\mu \mathrm{mol} / \mathrm{L})$, BAY 58-2667 $(0.001-10 \mu \mathrm{mol} / \mathrm{L})$, and NS1619 $(0.01-10 \mu \mathrm{mol} / \mathrm{L})$. For the relaxation curves, it typically took about 3 minutes for the vessel to reach a plateau at each dose for ACh and SNP, whereas for BAY 58-2667 it took 5-10 minutes depending on the concentration. Contractile response to potassium chloride $(\mathrm{KCl})(10$ $100 \mathrm{mmol} / \mathrm{L}), \mathrm{PGF}_{2 \alpha}(1-100 \mu \mathrm{mol} / \mathrm{L}), 5-\mathrm{HT}(0.01-10 \mu \mathrm{mol} / \mathrm{L})$, and endothelin 1 (ET-1) $(0.1-100 \mathrm{nmol} / \mathrm{L})$ was determined. For Rho kinase inhibition, aortic rings were preincubated with Y27632 $(1 \mu \mathrm{mol} / \mathrm{L})$ for 30 minutes and $\mathrm{ACh}$ relaxation was conducted. For oxidative stress experiments, aortic rings were preincubated with the superoxide scavenger Tempol $(1 \mathrm{mmol} / \mathrm{L})$ for 30 minutes, and ACh relaxation was conducted. Data were collected using PowerLab and analyzed using LabChart 5 software (AD Instruments).

Cerebral basilar arteries were cannulated onto glass pipettes and pressurized at $60 \mathrm{mmHg}$, and vascular reactivity experiments were conducted using a Danish Myograph Technology (DMT) system as previously reported $(11,16,24,58,59)$. Mice were euthanized as described above, and the basilar arteries were isolated, cleaned of connective tissues, and cannulated onto glass pipettes filled with oxygenated Krebs buffer in an organ chamber. Arteries were then transferred to a DMT pressurized myograph system and equilibrated for 30 minutes at 60 $\mathrm{mmHg}$ under no-flow conditions. Vessel integrity and viability were determined by $\mathrm{KCl}(100 \mathrm{mmol} / \mathrm{L})$. All arteries unresponsive to $\mathrm{KCl}$ were discarded. Arteries were precontracted with the thromboxane $\mathrm{A} 2$ mimetic (U46619) to 30\% internal diameter, and after a stable precontraction, concentration-response curves were determined for ACh $(0.001-100 \mu \mathrm{mol} / \mathrm{L})$ and SNP $(0.001-100 \mu \mathrm{mol} / \mathrm{L})$. For Rho kinase inhibition, basilar arteries were preincubated with Y27632 $(1 \mu \mathrm{mol} / \mathrm{L})$ for 30 minutes, and ACh relaxation was conducted.

Aortic stiffness measurements. PWV was determined as an index of arterial stiffness using the Indus Doppler Flow Velocity System (Indus Instruments) $(16,60)$. Briefly, mice were maintained under anesthesia ( $2 \%$ isoflurane $/ 1 \%$ oxygen) in a supine position, and core body temperature was kept constant at $37^{\circ} \mathrm{C}$. Continuous ECG recordings were collected from the paws, and pressure waveforms using a $20-\mathrm{MHz}$ probe were imaged at the descending aorta and abdominal aorta above the aortic bifurcation. Arrival and transit times were calculated over 5-7 cardiac cycles, and the distance between the descending and abdominal aorta was measured using a caliper. PWV was calculated as distance (meters) divided by the time (seconds). Pressure-diameter relationship studies were conducted as previously shown (61). Thoracic aortas were dissected free of adventitia adipose and cannulated onto steel cannulas in an organ bath containing calcium-free buffer supplemented with EGTA (2 $\mathrm{mmol} / \mathrm{L}$ ). The cannulated aortas were then pressurized from 0 to $200 \mathrm{mmHg}$, at $25-\mathrm{mmHg}$ increments, and inner and outer diameter as well as wall thickness were recorded using the DMT system as previously reported (16). A stress-strain relationship was determined, showing a downward shift indicative of arterial stiffness.

Aortic distensibility was determined as previously described (60). Adventitial collagen was stained and visualized using Masson's trichrome. As a direct biochemical measurement of total aortic collagen deposition, hydroxyproline content was determined using a biochemical assay. Elastin content was also determined as previously described (62). Briefly, insoluble elastin was separated from soluble proteins by incubation in $0.1 \mathrm{~N} \mathrm{NaOH}$ at $90^{\circ} \mathrm{C}$ for 45 minutes. The elastin fraction was further digested in $6 \mathrm{~N} \mathrm{HCl}$ at $105^{\circ} \mathrm{C}$ for 48 hours, neutralized, and then assayed for ninhydrin content.

Confocal microscopy. Thoracic aortas from $\mathrm{Cre}^{+} / C U L 3^{\text {llfl}}$ mice receiving either tamoxifen or corn oil as controls were dissected free of adventitial fat, embedded in OCT compound, and kept frozen at $-80^{\circ} \mathrm{C}$ until processed for sectioning. Aortas were sectioned at $10-\mu \mathrm{m}$ thickness and fixed with $4 \%$ paraformaldehyde for 1 hour at room temperature. Sections were rinsed with $1 \times$ PBS 3 times for 5 minutes. Slides were then permeabilized with $0.3 \%$ Triton X-100 in PBS for 30 minutes at room temperature and rinsed with $1 \times$ PBS 3 times for 5 minutes. Sections were then blocked with blocking buffer consisting of $10 \%$ goat serum, $0.3 \%$ Triton-X-100, and 1\% BSA in $1 \times$ PBS for 1 hour. Sections were incubated with primary antibody against Cullin-3 (catalog A301-109A, Bethyl Laboratories) diluted 1:100 or CD31 (catalog 553370, BD Biosciences) diluted 1:50 in blocking buffer overnight at $4^{\circ} \mathrm{C}$. Sections were rinsed with $1 \times$ PBS 3 times for 10 minutes each and were incubated with secondary antibody against Cullin-3 (anti-rabbit Alexa Fluor 568) and against CD31 (anti-rat Alexa Fluor 488) at a final dilution of 1:2000 for 2 hours. Sections were rinsed with 1× PBS 3 times for 10 minutes each. Sections were then mounted on glass slides with mounting medium containing DAPI (Vectashield, Vector Laboratories), and images were visualized using confocal microscopy (Zeiss LSM710).

Drugs and reagents. ACh, SNP, methacholine, DEA NONOate, KCl, NS1619, 5-HT, and Tempol were obtained from MilliporeSigma; and all were dissolved in saline. PGF ${ }_{2 \alpha}$ was from Pfizer; ET-1 was from Peninsula Laboratories and dissolved in water. U46619 and BAY 58-2667 were obtained from Cayman Chemical. 8-pCPT-cGMP was obtained from BioLog. 
Statistics. All data are expressed as mean \pm SEM. Data were analyzed using unpaired, 2-tailed Student's $t$ test, 1-way ANOVA, or 2-way ANOVA (repeated-measures when appropriate) using Tukey's or Bonferroni's post hoc test as indicated in each figure legend. $P \leq 0.05$ was considered statistically significant. Data were analyzed using GraphPad Prism 7 or 8 software.

Study approval. All animal protocols were approved by the University of Iowa and Medical College of Wisconsin Animal Care and Use Committees and were performed in accordance with the standards set forth by the NIH Guide for the Care and Use of Laboratory Animals (National Academies Press, 2011).

\section{Author contributions}

LNA generated $\mathrm{Cre}^{+} / C U L 3^{f / f l}$ mice and performed many of the experiments. JW contributed to the stress-strain experiments; ARN performed some vascular function studies on thoracic aortas; DRD performed surgeries to implant radiotelemeter devices; KTL performed quantitative PCR; HLK, JDS, and JAM performed data analysis. LNA, FWQ, and CDS conceived and supervised the project, performed data analysis, and wrote the manuscript. All authors analyzed the results, and read and approved the final version of the manuscript.

\section{Acknowledgments}

We thank Jeffrey Singer (Portland State University) for the gift of mice carrying a conditional allele of CUL3 $\left(C U L 3^{f / f f}\right)$. We also thank Isabella Grumbach (University of Iowa) for the gift of mice with inducible smooth muscle Cre driver. We thank William Paradee, Norma Sinclair, JoAnne Schwarting, and Patricia Yarolem for genotyping mice at the University of Iowa Gene Editing Core. We also thank Chantal Allamargot and Katherine Walters of the University of Iowa Central Microscopy Research Facility. This work was supported by the NIH (see below) and the University of Iowa. Its contents are solely the responsibility of the authors and do not necessarily represent the official views of the US National Institutes of Health or the University of Iowa. This work was supported through research grants from the NIH to CDS (HL084207, HL125603, HL131689, HL144807) and JAM (DK098141) and from the American Heart Association (AHA; 15SFRN23480000) to CDS. This work was supported in part by an Iowa Cardiovascular Interdisciplinary Research Fellowship (T32HL007121) to LNA and an AHA postdoctoral fellowship to JW (17POST33660685). The authors gratefully acknowledge the research support of the Roy J. Carver Trust.

Address correspondence to: Curt D. Sigmund, Department of Physiology, Medical College of Wisconsin, 573A BSB, 8701 Watertown Plank Road, Milwaukee, Wisconsin 53226-0509, USA. Phone: 414.955.8277; Email: csigmund@mcw.edu.

1. Kearney PM, Whelton M, Reynolds K, Muntner P, Whelton PK, He J. Global burden of hypertension: analysis of worldwide data. Lancet. 2005;365(9455):217-223.

2. Whelton PK, et al. 2017 ACC/AHA/AAPA/ABC/ACPM/AGS/APhA/ASH/ASPC/NMA/PCNA Guideline for the Prevention, Detection, Evaluation, and Management of High Blood Pressure in Adults: a report of the American College of Cardiology/American Heart Association Task Force on Clinical Practice Guidelines. Hypertension. 2018;71(6):e13-e115.

3. Acelajado MC, et al. Refractory hypertension: definition, prevalence, and patient characteristics. J Clin Hypertens (Greenwich). 2012;14(1):7-12.

4. Boyden LM, et al. Mutations in kelch-like 3 and cullin 3 cause hypertension and electrolyte abnormalities. Nature. 2012;482(7383):98-102.

5. Glover M, et al. Detection of mutations in KLHL3 and CUL3 in families with FHHt (familial hyperkalaemic hypertension or Gordon's syndrome). Clin Sci (Lond). 2014;126(10):721-726.

6. Louis-Dit-Picard H, et al. KLHL3 mutations cause familial hyperkalemic hypertension by impairing ion transport in the distal nephron. Nat Genet. 2012;44(4):456-460.

7. Tsuji S, et al. A young child with pseudohypoaldosteronism type II by a mutation of Cullin 3. BMC Nephrol. 2013;14:166.

8. Shibata S, Zhang J, Puthumana J, Stone KL, Lifton RP. Kelch-like 3 and Cullin 3 regulate electrolyte homeostasis via ubiquitination and degradation of WNK4. Proc Natl Acad Sci U S A. 2013;110(19):7838-7843.

9. Wakabayashi M, et al. Impaired KLHL3-mediated ubiquitination of WNK4 causes human hypertension. Cell Rep. 2013;3(3):858-868.

10. Halabi CM, et al. Interference with PPAR gamma function in smooth muscle causes vascular dysfunction and hypertension. Cell Metab. 2008;7(3):215-226.

11. Pelham CJ, et al. Cullin-3 regulates vascular smooth muscle function and arterial blood pressure via PPAR $\gamma$ and RhoA/Rho-kinase. Cell Metab. 2012;16(4):462-472.

12. Chen Y, et al. Cullin mediates degradation of RhoA through evolutionarily conserved BTB adaptors to control actin cytoskeleton structure and cell movement. Mol Cell. 2009;35(6):841-855. 
13. Ibeawuchi SR, Agbor LN, Quelle FW, Sigmund CD. Hypertension-causing mutations in Cullin3 protein impair RhoA protein ubiquitination and augment the association with substrate adaptors. J Biol Chem. 2015;290(31):19208-19217.

14. McCormick JA, et al. Hyperkalemic hypertension-associated cullin 3 promotes WNK signaling by degrading KLHL3. JClin Invest. 2014;124(11):4723-4736.

15. Schumacher FR, et al. Characterisation of the Cullin-3 mutation that causes a severe form of familial hypertension and hyperkalaemia. EMBO Mol Med. 2015;7(10):1285-1306.

16. Agbor LN, et al. Cullin-3 mutation causes arterial stiffness and hypertension through a vascular smooth muscle mechanism. JCI Insight. 2016;1(19):e91015.

17. Ferdaus MZ, et al. Mutant Cullin 3 causes familial hyperkalemic hypertension via dominant effects. JCI Insight. 2017;2(24):e96700.

18. Genschik P, Sumara I, Lechner E. The emerging family of CULLIN3-RING ubiquitin ligases (CRL3s): cellular functions and disease implications. EMBO J. 2013;32(17):2307-2320.

19. Petroski MD, Deshaies RJ. Function and regulation of cullin-RING ubiquitin ligases. Nat Rev Mol Cell Biol. 2005;6(1):9-20.

20. Xu L, et al. BTB proteins are substrate-specific adaptors in an SCF-like modular ubiquitin ligase containing CUL-3. Nature. 2003;425(6955):316-321.

21. Zhang DD, Lo SC, Cross JV, Templeton DJ, Hannink M. Keap1 is a redox-regulated substrate adaptor protein for a Cul3-dependent ubiquitin ligase complex. Mol Cell Biol. 2004;24(24):10941-10953.

22. Mukohda M, et al. RhoBTB1 protects against hypertension and arterial stiffness by restraining phosphodiesterase 5 activity. J Clin Invest. 2019;130:2318-2332.

23. Wimuttisuk W, Singer JD. The Cullin3 ubiquitin ligase functions as a Nedd8-bound heterodimer. Mol Biol Cell. 2007;18(3):899-909.

24. De Silva TM, et al. Role of peroxisome proliferator-activated receptor- $\gamma$ in vascular muscle in the cerebral circulation. Hypertension. 2014;64(5):1088-1093.

25. Beyer AM, et al. Interference with PPAR $\gamma$ signaling causes cerebral vascular dysfunction, hypertrophy, and remodeling. Hypertension. 2008;51(4):867-871.

26. Beyer AM, et al. Endothelium-specific interference with peroxisome proliferator activated receptor gamma causes cerebral vascular dysfunction in response to a high-fat diet. Circ Res. 2008;103(6):654-661.

27. Wallace SM, et al. Isolated systolic hypertension is characterized by increased aortic stiffness and endothelial dysfunction. Hypertension. 2007;50(1):228-233.

28. Ruetten H, Zabel U, Linz W, Schmidt HH. Downregulation of soluble guanylyl cyclase in young and aging spontaneously hypertensive rats. Circ Res. 1999;85(6):534-541.

29. Schäfer A, et al. Increased cytochrome P4502E1 expression and altered hydroxyeicosatetraenoic acid formation mediate diabetic vascular dysfunction: rescue by guanylyl-cyclase activation. Diabetes. 2010;59(8):2001-2009.

30. Klöss S, Rodenbach D, Bordel R, Mulsch A. Human-antigen R (HuR) expression in hypertension: downregulation of the mRNA stabilizing protein HuR in genetic hypertension. Hypertension. 2005;45(6):1200-1206.

31. Kloss S, Furneaux H, Mülsch A. Post-transcriptional regulation of soluble guanylyl cyclase expression in rat aorta. J Biol Chem. 2003;278(4):2377-2383.

32. Cabilla JP, Nudler SI, Ronchetti SA, Quinteros FA, Lasaga M, Duvilanski BH. Nitric oxide-sensitive guanylyl cyclase is differentially regulated by nuclear and non-nuclear estrogen pathways in anterior pituitary gland. PLoS ONE. 2011;6(12):e29402.

33. de Frutos S, Nitta CH, Caldwell E, Friedman J, Gonzalez Bosc LV. Regulation of soluble guanylyl cyclase-alpha1 expression in chronic hypoxia-induced pulmonary hypertension: role of NFATc3 and HuR. Am J Physiol Lung Cell Mol Physiol. 2009;297(3):L475-L486.

34. Rahaman MM, et al. Cytochrome b5 reductase 3 modulates soluble guanylate cyclase redox state and cGMP signaling. Circ Res. 2017;121(2):137-148

35. Ketsawatsomkron $P$, et al. PPAR $\gamma$ regulates resistance vessel tone through a mechanism involving RGS5-mediated control of PKC and BKCa channel activity. Circ Res. 2012;111(11):1446-1458.

36. Cornelius RJ, et al. Dual gain and loss of cullin 3 function mediates familial hyperkalemic hypertension. Am J Physiol Renal Physiol. 2018;315(4):F1006-F1018.

37. Abdel Khalek W, et al. Severe arterial hypertension from Cullin 3 mutations is caused by both renal and vascular effects. $J A m$ Soc Nephrol. 2019;30(5):811-823.

38. Panza JA, Quyyumi AA, Brush JE Jr, Epstein SE. Abnormal endothelium-dependent vascular relaxation in patients with essential hypertension. N Engl J Med. 1990;323(1):22-27.

39. Huang PL, et al. Hypertension in mice lacking the gene for endothelial nitric oxide synthase. Nature. 1995;377(6546):239-242.

40. Shesely EG, et al. Elevated blood pressures in mice lacking endothelial nitric oxide synthase. Proc Natl Acad Sci USA. 1996;93(23):13176-13181.

41. Friebe A, Mergia E, Dangel O, Lange A, Koesling D. Fatal gastrointestinal obstruction and hypertension in mice lacking nitric oxide-sensitive guanylyl cyclase. Proc Natl Acad Sci U S A. 2007;104(18):7699-7704.

42. Buys ES, et al. Gender-specific hypertension and responsiveness to nitric oxide in sGCalpha1 knockout mice. Cardiovasc Res. 2008;79(1):179-186.

43. Groneberg D, König P, Wirth A, Offermanns S, Koesling D, Friebe A. Smooth muscle-specific deletion of nitric oxide-sensitive guanylyl cyclase is sufficient to induce hypertension in mice. Circulation. 2010;121(3):401-409.

44. Galley JC, et al. Antagonism of forkhead box subclass $\mathrm{O}$ transcription factors elicits loss of soluble guanylyl cyclase expression. Mol Pharmacol. 2019;95(6):629-637.

45. Stasch JP, et al. Targeting the heme-oxidized nitric oxide receptor for selective vasodilatation of diseased blood vessels. $J$ Clin Invest. 2006;116(9):2552-2561.

46. Burgoyne JR, Prysyazhna O, Richards DA, Eaton P. Proof of principle for a novel class of antihypertensives that target the oxidative activation of PKG I $\alpha$ (protein kinase G I $\alpha$ ). Hypertension. 2017;70(3):577-586.

47. Schiffrin EL, Larivière R, Li JS, Sventek P, Touyz RM. Deoxycorticosterone acetate plus salt induces overexpression of vascular endothelin-1 and severe vascular hypertrophy in spontaneously hypertensive rats. Hypertension. 1995;25(4 pt 2):769-773. 
48. van Gorp AW, Schenau DS, Hoeks AP, Boudier HA, de Mey JG, Reneman RS. In spontaneously hypertensive rats alterations in aortic wall properties precede development of hypertension. Am J Physiol Heart Circ Physiol. 2000;278(4):H1241-H1247.

49. Laurent S, et al. Aortic stiffness is an independent predictor of all-cause and cardiovascular mortality in hypertensive patients. Hypertension. 2001;37(5):1236-1241.

50. Jung SM, et al. Increased tissue transglutaminase activity contributes to central vascular stiffness in eNOS knockout mice. Am J Physiol Heart Circ Physiol. 2013;305(6):H803-H810.

51. De Nicola L, Blantz RC, Gabbai FB. Nitric oxide and angiotensin II. Glomerular and tubular interaction in the rat. J Clin Invest. 1992;89(4):1248-1256

52. Zeniya M, et al. Dietary salt intake regulates WNK3-SPAK-NKCC1 phosphorylation cascade in mouse aorta through angiotensin II. Hypertension. 2013;62(5):872-878.

53. Bergaya $\mathrm{S}$, et al. WNK1 regulates vasoconstriction and blood pressure response to $\alpha$ 1-adrenergic stimulation in mice. Hypertension. 2011;58(3):439-445.

54. Park HW, et al. Serine-threonine kinase with-no-lysine 4 (WNK4) controls blood pressure via transient receptor potential canonical 3 (TRPC3) in the vasculature. Proc Natl Acad Sci U S A. 2011;108(26):10750-10755

55. Ray JL, Leach R, Herbert JM, Benson M. Isolation of vascular smooth muscle cells from a single murine aorta. Methods Cell Sci. 2001;23(4):185-188.

56. Nair AR, et al. Interference with endothelial PPAR (peroxisome proliferator-activated receptor)- $\gamma$ causes accelerated cerebral vascular dysfunction in response to endogenous renin-angiotensin system activation. Hypertension. 2018;72(5):1227-1235.

57. Livak KJ, Schmittgen TD. Analysis of relative gene expression data using real-time quantitative PCR and the 2(-delta delta C(T)) method. Methods. 2001;25(4):402-408.

58. Hu C, et al. Retinol-binding protein 7 is an endothelium-specific PPAR $\gamma$ cofactor mediating an antioxidant response through adiponectin. JCI Insight. 2017;2(6):e91738

59. Hu C, Lu KT, Mukohda M, Davis DR, Faraci FM, Sigmund CD. Interference with PPAR $\gamma$ in endothelium accelerates angiotensin II-induced endothelial dysfunction. Physiol Genomics. 2016;48(2):124-134.

60. Bersi MR, Bellini C, Wu J, Montaniel KRC, Harrison DG, Humphrey JD. Excessive adventitial remodeling leads to early aortic maladaptation in angiotensin-induced hypertension. Hypertension. 2016;67(5):890-896.

61. Wu J, et al. Inflammation and mechanical stretch promote aortic stiffening in hypertension through activation of p38 mitogen-activated protein kinase. Circ Res. 2014;114(4):616-625

62. Hofman K, Hall B, Cleaver H, Marshall S. High-throughput quantification of hydroxyproline for determination of collagen Anal Biochem. 2011;417(2):289-291. 